\title{
High MUC2 Mucin Expression and Misfolding Induce Cellular Stress, Reactive Oxygen Production, and Apoptosis in Goblet Cells
}

\author{
Adelaide Tawiah, Steve Cornick, France Moreau, Hayley Gorman, Manish Kumar, Sameer Tiwari, and Kris Chadee
}

From the Department of Microbiology, Immunology and Infectious Diseases, Gastrointestinal Research Group, Snyder Institute for Chronic Diseases, Faculty of Medicine, University of Calgary Health Sciences Centre, Calgary, Alberta, Canada

Accepted for publication

February 20, 2018.

Address correspondence to Kris Chadee, Ph.D., Department of Microbiology, Immunology and Infectious Diseases, University of Calgary Health Sciences Centre, 3330 Hospital Dr NW, Calgary, AB, Canada T2N 4N1. E-mail: kchadee@ ucalgary.ca.

\begin{abstract}
MUC2 mucin is a large glycoprotein produced by goblet cells that forms the protective mucus blanket overlying the intestinal epithelium as the first line of innate host defense. High MUC2 production in inflammatory bowel disease and infectious colitis depletes goblet cells and the mucus layer by an unknown mechanism. Herein, we analyzed the effect of high MUC2 biosynthesis on endoplasmic reticulum (ER) stress and apoptosis in goblet cells using a high MUC2-producing human goblet cell line (HT29-H) and an HT29-H clone (HT29-L) silenced for MUC2 expression by lentivirus-mediated shRNA. Goblet cell ER stress and apoptosis were quantified during early onset of dextran sulfate sodiuminduced colitis in C57BL/ 6 and Math $1^{\text {M1GFP }}$ mice. Compared with HT29-L and MUC2 nonproducing Caco-2 cells, high MUC2-producing HT29-H cells had significantly increased ER stress and apoptosis after treatment with ER stress-inducing agents. Apoptosis was driven by increased misfolded MUC2 that triggered elevated levels of reactive oxygen species. Correcting MUC2 folding and inhibiting reactive oxygen species alleviated ER stress and rescued cells from apoptosis. During early-onset colitis, mucus hypersecretion caused severe ER stress and apoptosis of goblet cells that preceded absorptive epithelial cell damage. Thus, in gastrointestinal inflammation, high MUC2 biosynthesis and goblet cell apoptosis lead to a dysfunctional epithelial barrier. Enhancing MUC2 folding may help alleviate goblet cell depletion and maintain mucosal integrity. (Am J Pathol 2018, 188: 1354-1373; https://doi.org/ 10.1016/j.ajpath.2018.02.007)
\end{abstract}

The gastrointestinal mucosal barrier is made up of a single layer of epithelial cells, immune cells, and resident microbiota. ${ }^{1}$ The surface of the epithelium is covered with a thick viscous layer of mucus, produced by goblet cells, which, together with antimicrobial peptides, constitutes the frontline of innate host defense. ${ }^{2}$ Colonic mucus is organized in two layers: an inner layer, which is firmly adherent to epithelial cells and impermeable to enteric bacteria; and an outer layer, colonized by resident microbiota. ${ }^{3}$ Mucus forms a physical barrier between gut contents and the underlying epithelium, preventing entry of noxious substances and enteric pathogens into the body. ${ }^{4}$ The primary constituent of mucus is highmol. wt. mucin glycoproteins. Mucins are broadly classified into two groups: secreted mucins and transmembrane mucins. ${ }^{5}$ Secreted mucins contribute to the formation of the mucus gel, whereas the transmembrane mucins form the glycocalyx and are thought to be involved in signaling events and barrier defense. ${ }^{6}$ The stomach and salivary glands secrete MUC5AC and MUC6 and MUC5B, respectively, whereas in the intestines, MUC2 is the major gel-forming mucin. ${ }^{6}$ The intestines also produce the transmembrane mucins, MUC1, MUC3, MUC4, MUC12, MUC13, and MUC17.

MUC2 is a large glycoprotein with approximately 5179 amino acids. It contains a large central $\mathrm{O}$-glycosylated domain and $\mathrm{N}$ - and C-terminal cysteine-rich domains that homooligomerize to form the complex mucin structure. ${ }^{8}$ Assembly of MUC2 is a complex process that starts in the endoplasmic

Supported by Canadian Institutes of Health Research grant KC MOP142776, Crohn's and Colitis Canada (K.C.), and the Eleanor Mackie Doctoral Scholarship in Women's Health (A.T.).

Disclosures: None declared. 
reticulum (ER), where MUC2 undergoes N-glycosylation and dimerizes via intermolecular disulfide bonding between the C-terminal cysteine knot domains. ${ }^{9}$ In the Golgi apparatus, it becomes extensively $\mathrm{O}$-glycosylated and undergoes trimerization in the $\mathrm{N}$-terminal region, resulting in the formation of large polymers. ${ }^{10}$ The fully glycosylated and processed MUC2 mucin is densely packed, stored in secretory granules/ vesicles, and released constitutively or in a stimulated manner. ${ }^{11}$ The proper structure of MUC2 is critical in maintaining the mucus barrier, and alterations in its structure damage the mucus gel that facilitates penetration and absorption of toxic substances, resulting in inflammation. ${ }^{12}$

Inflammatory bowel disease (IBD), which is broadly grouped into Crohn disease and ulcerative colitis, is a chronic and relapsing gastrointestinal tract inflammatory disorder whose etiology is unknown. A major hallmark of IBD is goblet cell pathology, typically described as goblet cell depletion. In ulcerative colitis, the mucus layer is thin because of goblet cell depletion and smaller goblet cell thecae. ${ }^{13}$ Although the mechanism of goblet cell depletion is unknown, the small goblet cell theca contains fewer mucin granules that are filled with nonglycosylated MUC2 precursors. ${ }^{14}$ These partially synthesized or unfolded MUC2 mucins accumulate within the ER and cause ER stress. In IBD, ER stress in goblet cells has been associated with either MUC2 mutations or deficiencies of MUC2 folding enzymes. ${ }^{14-16}$ During ER stress, a series of reactions, known as the unfolded protein response, is activated to restore correct protein folding and ER homeostasis. ${ }^{17}$ When stress is prolonged and ER homeostasis is not achieved, the goal of the unfolded protein response changes from that of a cell survival mechanism to that which commits the cell to death by activating proapoptotic proteins, such as CCAATenhancer-binding protein homologous protein (CHOP). ${ }^{18}$

Under basal conditions, goblet cells continually synthesize and secrete MUC2 to replenish the mucus layer covering the epithelium. ${ }^{19}$ However, when goblet cells are exposed to mucin secretagogues, pathogens, and other injurious substances during inflammation, there is accelerated biosynthesis and secretion of MUC2. It is known that MUC2 mutations and deficiencies in mucin-folding enzymes lead to inflammation attributable to ER stress. However, there is little information on how MUC2 production is regulated and whether goblet cells undergo increased ER stress and apoptosis in response to high MUC2 production and secretion during disease. In this study, we showed that high MUC2 production increases goblet cell susceptibility to ER stress and apoptosis that abrogated innate host defenses to accelerate the onset of colitis.

\section{Materials and Methods}

\section{Ethics Statement}

All studies were approved under the University of Calgary (Calgary, Alberta, Canada) Animal Care Committee, which adheres to the principles and policies on Guide to the Care and Use of Experimental Animals by the Canadian Council on Animal Care.

\section{Generation of HT29-H and HT29-L Cell Lines}

HT29-H and HT29-L are high and low MUC2-producing human colonic adenocarcinoma cell lines, respectively. The HT29-H cells used in this study were a gift from Dr. Christian Laboisse (Université de Nantes, Nantes, France). These are polarized permanently differentiated HT29 cell clones (CL16E) ${ }^{20}$ that highly express MUC2 mucin. The cells are locked in their phenotype and do not continually differentiate in culture media. Briefly, these cells were produced by culturing the parental wild-type HT29 cells in the presence of $5 \mathrm{mmol} / \mathrm{L}$ sodium butyrate for 9 days and then subculturing into media containing sodium butyrate for another 14 days. The cells were then cultured in a standard medium without sodium butyrate. During this period, the cells differentiated into a goblet-like phenotype with the ability to produce large amounts of mucus. ${ }^{20}$ HT29$\mathrm{L}$ is a clone of HT29-H with MUC2 silenced using lentivirus shRNA. Lentivirus-based silencing of genes results in stable gene knockdown as the virus integrates into the genome. Hairpin MUC2 shRNA sequences composed of a 21-bp stem and a 6-bp loop were cloned into pLKO.1 viral vectors and cotransfected into packaging cells, HEK293T, along with an envelope and a packaging plasmid. Viral particles containing the transfected shRNA plasmid released in culture after 24 hours were collected and concentrated by ultracentrifugation. To achieve maximal MUC2 silencing, the viral particles were transduced into HT29-H cells in the presence of polybrene. Transduced cells were selected with puromycin. HT29-H cells also contain the empty lentiviral vector.

\section{HT29-H/L Cell Culture and Treatment}

HT29-H and HT29-L cells were cultured in Dulbecco's modified Eagle's medium supplemented with $10 \%$ (v/v) heat-inactivated fetal bovine serum, penicillin/streptomycin $(100 \mathrm{U} / \mathrm{mL} / 100 \mathrm{mg} / \mathrm{mL})$, and $1 \mathrm{mmol} / \mathrm{L}$ sodium pyruvate (all from Invitrogen, Burlington, $\mathrm{ON}, \mathrm{Canada}$ ), at $37^{\circ} \mathrm{C}$ in $5 \% \mathrm{CO}_{2}$. The cells were seeded at $1 \times 10^{5}$ cells $/ \mathrm{mL}$ in 12-well plates, and experiments were performed when cells were approximately $80 \%$ confluent (day 6). Basal expression of the stress marker GRP76 mRNA did not change significantly from days 4 to 8 in culture (Supplemental Figure S1). The cells were treated with the following chemicals: staurosporine (Calbiochem, San Diego, CA), tumor necrosis factor- $\alpha$, interferon- $\gamma$ (Peprotech, Rocky Hill, NJ), and tunicamycin (MP Biomedicals, Burlington, ON, Canada) at $10 \mu \mathrm{g} / \mathrm{mL}$, tauroursodeoxycholic acid (TUDCA; EMD Millipore, Calbiochem, Etobicoke, ON, Canada) at $1 \mathrm{mmol} / \mathrm{L}$, and diphenyleneiodonium (DPI; Sigma-Aldrich, Oakville, ON, Canada) at $10 \mu \mathrm{mol} / \mathrm{L}$. 


\section{Mucin Isolation}

HT29-H/L cells were cultured in 6-well plates to confluence, and mucins (insoluble form) were extracted with guanidinium hydrochloride extraction buffer $(6 \mathrm{~mol} / \mathrm{L}$ guanidinium hydrochloride, $5 \mathrm{mmol} / \mathrm{L}$ EDTA, and $10 \mathrm{mmol} / \mathrm{L} \mathrm{NaH} \mathrm{PO}_{4}$, $\mathrm{pH}$ 6.5). The insoluble mucins were pelleted by centrifugation for 20 minutes at $30,000 \times g$, followed by six washes in
A

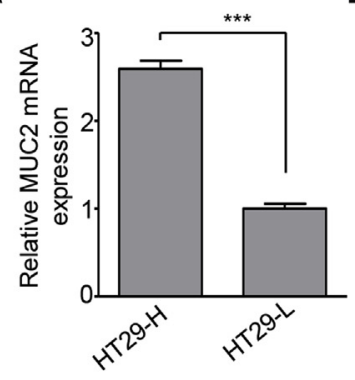

$\mathbf{E}$

Cleaved PARP
B

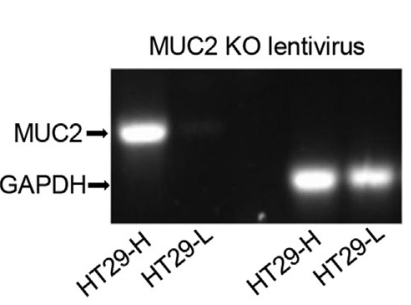

C

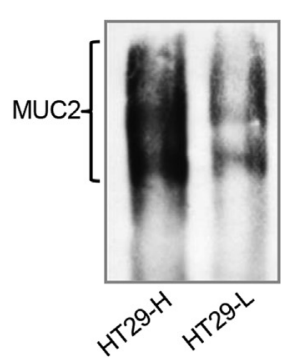

D

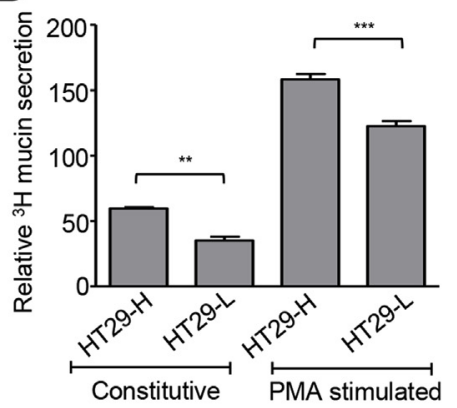

CASP3
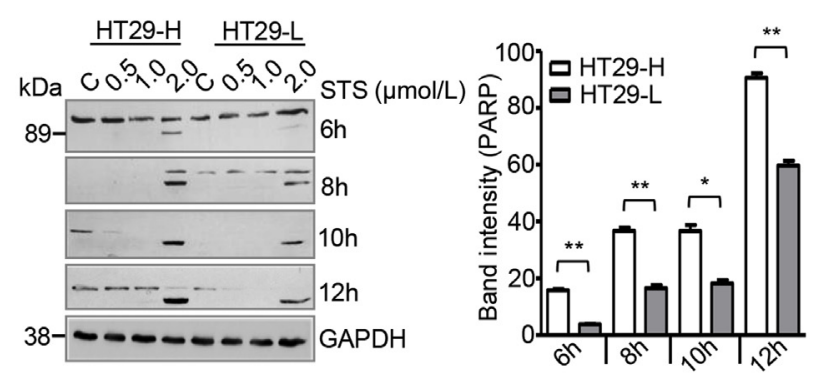

HT29-H HT29-L

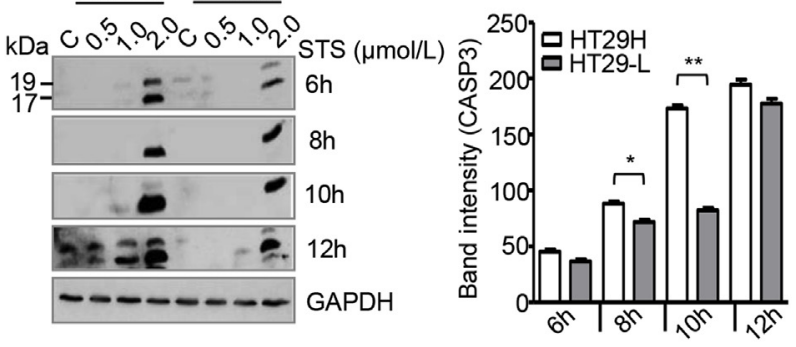

$\mathbf{F}$
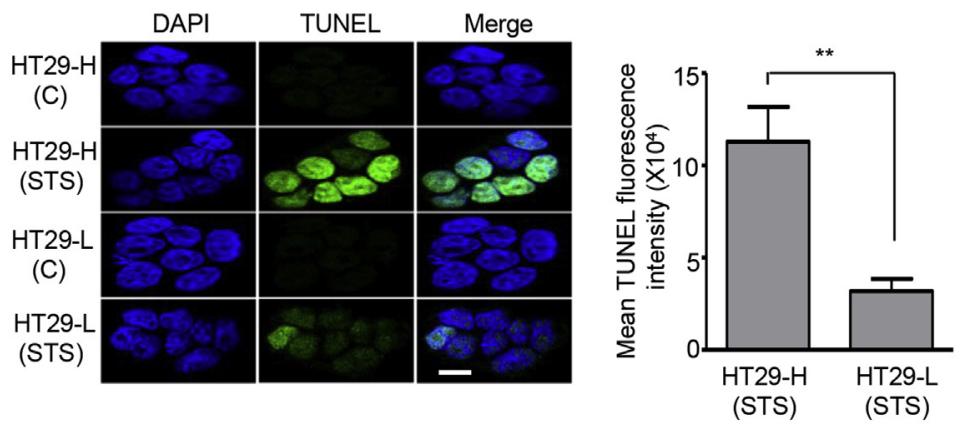

H
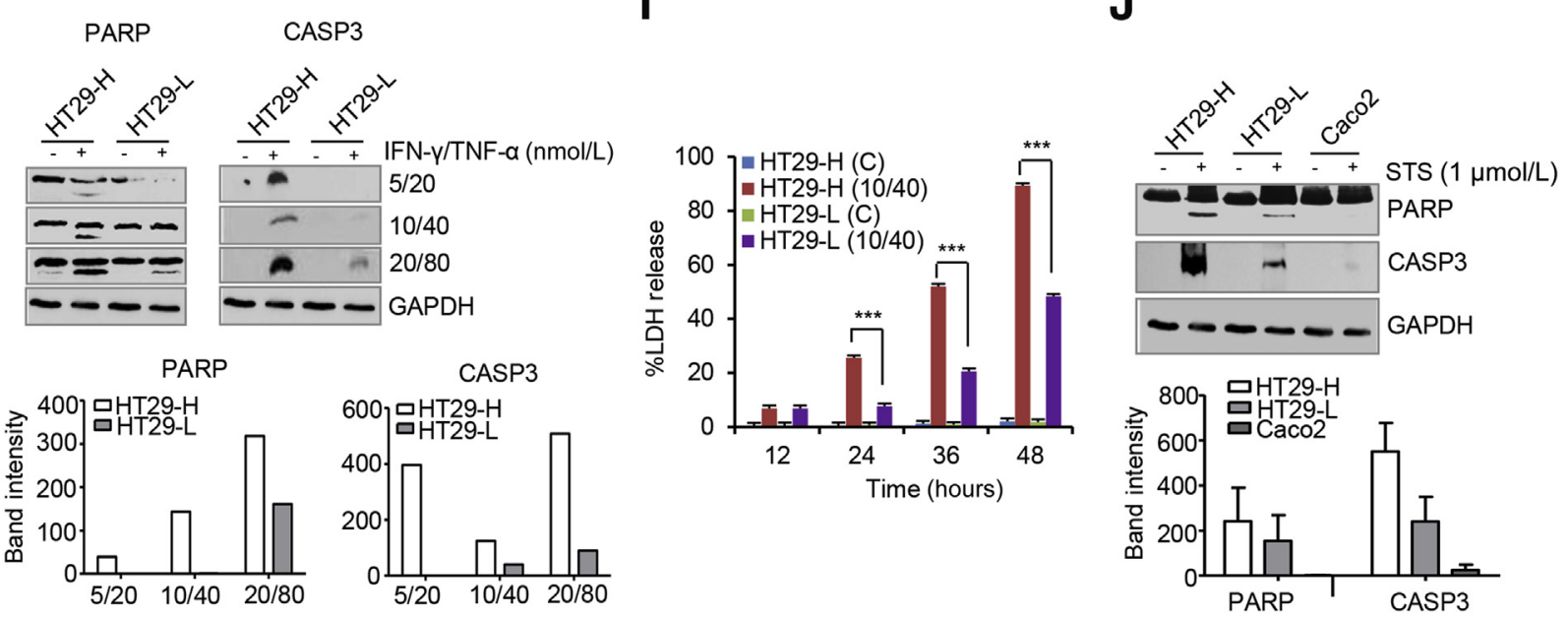
guanidinium hydrochloride extraction buffer and six washes in Dulbecco's phosphate-buffered saline (PBS). The mucins were extensively dialyzed against distilled water overnight and lyophilized for 18 to 24 hours until completely dried. The lyophilized samples were reconstituted in $0.01 \mathrm{~mol} / \mathrm{L}$ Tris buffer and purified using a Sepharose 4B column, which separates high-mol. wt. mucins (in void volume; blue dextran) from nonmucin components (bovine serum albumin and B12 mol. wt. markers). Proteins eluted in the void volume were pooled, further dialyzed and lyophilized, and purified using a cesium chloride density gradient. The protein samples were separated on an agarose gel and immunoblotted using human MUC2 antibody, generated in rabbits against cesium chloride-purified LS $174 \mathrm{~T}$ cell MUC2, ${ }^{21}$ to show amount of mature MUC2 in HT29-H and HT29-L cells. To determine nonglycosylated MUC2, HT29-H/L cells were lysed with lysis buffer $(1 \mathrm{mmol} / \mathrm{L}$ EDTA, $10 \mathrm{mmol} / \mathrm{L}$ Tris-HCl, $10 \%$ SDS, $5 \mathrm{mmol} / \mathrm{L} \mathrm{NaCl}, 20 \%$ Triton X-100, phenylmethylsulfonyl fluoride, and a protease inhibitor cocktail; Sigma-Aldrich) after medium was removed. The samples were separated on a $1 \%$ agarose gel, Western blotted onto a nitrocellulose membrane, and probed with a MUC2 precursor antibody (4F1), which detects sequences in the tandem repeat of MUC2, to detect non-glycosylated MUC2. ${ }^{22}$ The 4F1 monoclonal antibody for the detection of non-glycosylated MUC2 precursor was a kind gift from Professor Michael McGuckin (University of Queensland, South Brisbane, QLD, Australia).

\section{Mucin Secretion}

HT29-H/L cells were metabolically labeled with ${ }^{3} \mathrm{H}$-glucosamine for 48 hours and then treated with $20 \mathrm{mmol} / \mathrm{L}$ phorbol myristate acetate for 3 hours or left untreated. After treatment, the cell culture supernatants were precipitated with equal volumes of $10 \%$ trichloroacetic acid and $1 \%$ phosphotungstic acid overnight at $4{ }^{\circ} \mathrm{C}$ to recover the mucins. Mucins were solubilized in water and neutralized to $\mathrm{pH} 7.0$ to 7.4 with $0.1 \mathrm{~mol} / \mathrm{L} \mathrm{NaOH}$. Scintillation cocktail (5 mL; UniverSol; MP Biochemicals, Santa Ana, CA) was added, and ${ }^{3} \mathrm{H}$-activity (a measure of mucus secretion) was determined in a scintillation counter. ${ }^{3} \mathrm{H}$-glucosamine-labelled mucin secretion was confirmed in the void volume by Sepharose 4B column (GE Healthcare Bio-Sciences, Issaquah, WA) chromatography.

\section{RNA Isolation and Gene Expression Analysis}

Total RNA from cells and full-thickness colonic tissues were extracted with TRIzol reagent (Invitrogen; Life Technologies, Carlsbad, CA), according to the manufacturer's instructions. RNA quality and yield were assessed using NANODROP1000 spectrophotometer (Thermo Fisher Scientific, Wilmington, DE). RNA ( $1 \mu \mathrm{g})$ was reverse transcribed to cDNA using random hexamer primers and Moloney murine leukemia virus reverse transcriptase (Life Technologies, Burlington, ON, Canada). For real-time quantitative PCR, each reaction mixture contained $1 \mu \mathrm{L}$ cDNA, used with SYBR Green master mix (Invitrogen), and $100 \mu \mathrm{mol} / \mathrm{L}$ primers in a final reaction volume of $20 \mu \mathrm{L}$. Real-time quantitative PCR was performed on a Corbett Rotor Gene 3000 system (Qiagen, Valencia, CA), and data were analyzed using the $2^{-\Delta \Delta C T}$ method. Expression of mRNA was investigated using the following primers: $5^{\prime}$ GCCTGTATTTCTAGACCTGCC- $3^{\prime}$ (forward) and $5^{\prime}$ TTCATCTTGCCAGCCAGTTG-3' (reverse) for human GRP78; 5'-GCACTGACTGCCAGAGATCG-3' (forward) and $5^{\prime}$-GCGCTGTCTTAACTCCTGGT- $3^{\prime}$ (reverse) for human sXBP1; 5'-TGATGACATCAAGAAGGTGGTGAAG-3' (forward) and $5^{\prime}$-TCCTTGGAGGCCATGTGGGCCAT-3' (reverse) for human glyceraldehyde-3-phosphate dehydrogenase; 5'-CAGCACCGATTGCTGAGTTG-3' (forward) and 5'-GCTGGTCATCTCAATGGCAG-3' (reverse) for human MUC2; 5'-AGTTCGACTTGGATGCCCTG-3' (forward) and 5'-CCAACGTGGTCAGAAGGTCA-3' (reverse) for human ATF4; 5'-GCGCATGAAGGAGAAA-GAAC-3' (forward) and 5'-CCAATTGTTCATGCTTGGTG-3' (reverse) for human CHOP; 5'-GTTGGGGTGACCAACTCAT-3' (forward) and 5'-GTGGGCACTCATCCAAGTGA-3' (reverse) for human anterior gradient 2; 5'-TTGCTACTCAAGCCCCACAG-3' (forward) and 5'CCGGATGATGCCCTTCTTGT-3' (reverse) for human SPDEF; $5^{\prime}$-TGCTGCTAGGCCTGCTCCGA- $3^{\prime}$ (forward) and $5^{\prime}$-CGACCACCGTGCCCACATCC-3' (reverse) for mouse GRP78; 5'-TGCTGTCTGCCGGTTTAAGT-3' (forward) and

Figure 1 High MUC2-producing HT29-H cells are more susceptible to apoptosis compared with HT29-L cells. A: Total mRNA was extracted from HT29-H/L cells, and MUC2 gene expression was quantified by real-time quantitative PCR. MUC2 expression was normalized to GAPDH as housekeeping gene. B: Agarose gel electrophoresis of PCR products. C: Whole cellular mucin protein was isolated from HT29-H/L cells by guanidinium hydrochloride extraction, separated on an agarose gel, and immunoblotted using human MUC2 antibodies that detect mature mucin. D: Cells were metabolically labeled with ${ }^{3} \mathrm{H}-\mathrm{glucosamine}$ for 48 hours (h) to allow glycosylation of MUC2 glycoproteins and then stimulated with $20 \mathrm{mmol} / \mathrm{L}$ phorbol myristate acetate (PMA) for 3 hours. The ${ }^{3} \mathrm{H}$-radiolabeled newly synthesized glycoproteins were fractionated by Sepharose $4 \mathrm{~B}$ column chromatography, and ${ }^{3} \mathrm{H}$ activity was determined in a scintillation counter. Only high relative molecular mass void volume mucin from the Sepharose 4B column is depicted in the histogram. E: HT29-H/L cells were left untreated or stimulated with different concentrations of staurosporine (STS) at various time points. Poly (ADP-ribose) polymerase (PARP) cleavage and caspase 3 activation (CASP3) were analyzed by Western blotting, and densitometric analysis of the $2 \mu \mathrm{mol} / \mathrm{L}$ band on the Western blot was performed. F: Cells were treated with $1 \mu \mathrm{mol} / \mathrm{L}$ STS for 12 hours, and DNA fragmentation was assessed using terminal deoxynucleotidyl transferase-mediated dUTP nick-end labeling (TUNEL) assay. Images were obtained using confocal microscopy, and fluorescence intensity was quantified. G: Lactose dehydrogenase (LDH) release (marker for cell death) was measured by spectrophotometric absorbance at $490 \mathrm{~nm}$ after treating cells with $2 \mu \mathrm{mol} / \mathrm{L} \mathrm{STS}$. H: HT29-H/L cells were treated with different concentrations of interferon (IFN)- $\gamma /$ tumor necrosis factor (TNF)- $\alpha$ for 12 hours, and apoptosis was analyzed by Western blot. I: LDH release was measured by spectrophotometric absorbance at $490 \mathrm{~nm}$ after stimulation with $20 / 80 \mu \mathrm{mol} / \mathrm{L} \mathrm{IFN}-\gamma / T N F-\alpha$. J: Cells were treated with $1 \mu \mathrm{mol} / \mathrm{L}$ STS for 12 hours, and PARP and caspase 3 cleavages were analyzed by Western blotting. Data are expressed as means \pm SEM $(\mathbf{A}, \mathbf{D}-\mathbf{G}, \mathbf{I}) . n=3(\mathbf{A}, \mathbf{D}-\mathbf{G}, \mathbf{I})$. ${ }^{*} P<0.05, * * P<0.01$, and $* * * P<0.001$ (t-test). Scale bar $=10 \mu \mathrm{m}(\mathbf{F}) . \mathrm{C}$, control; h, hour; K0, knockout. 
A
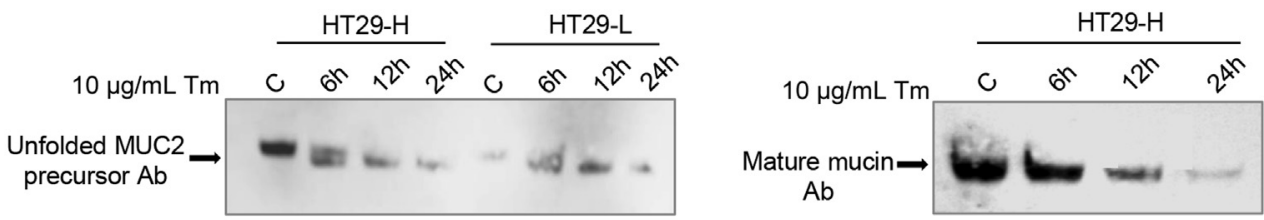

B

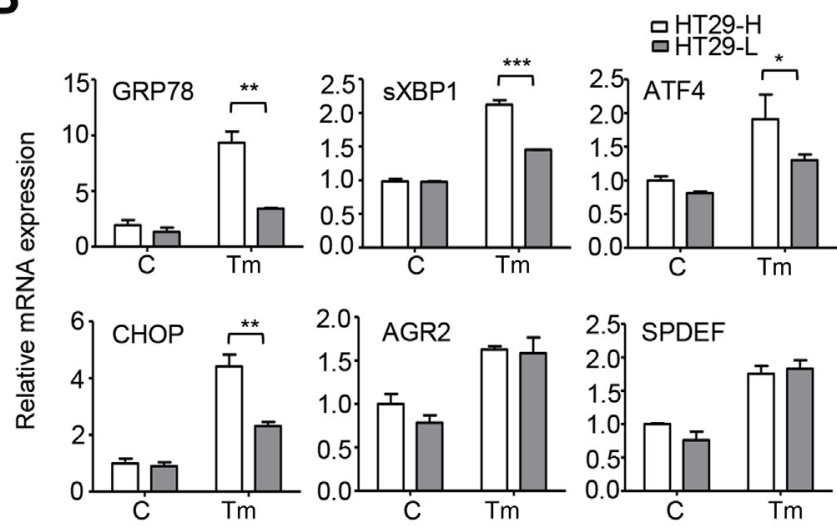

C

D
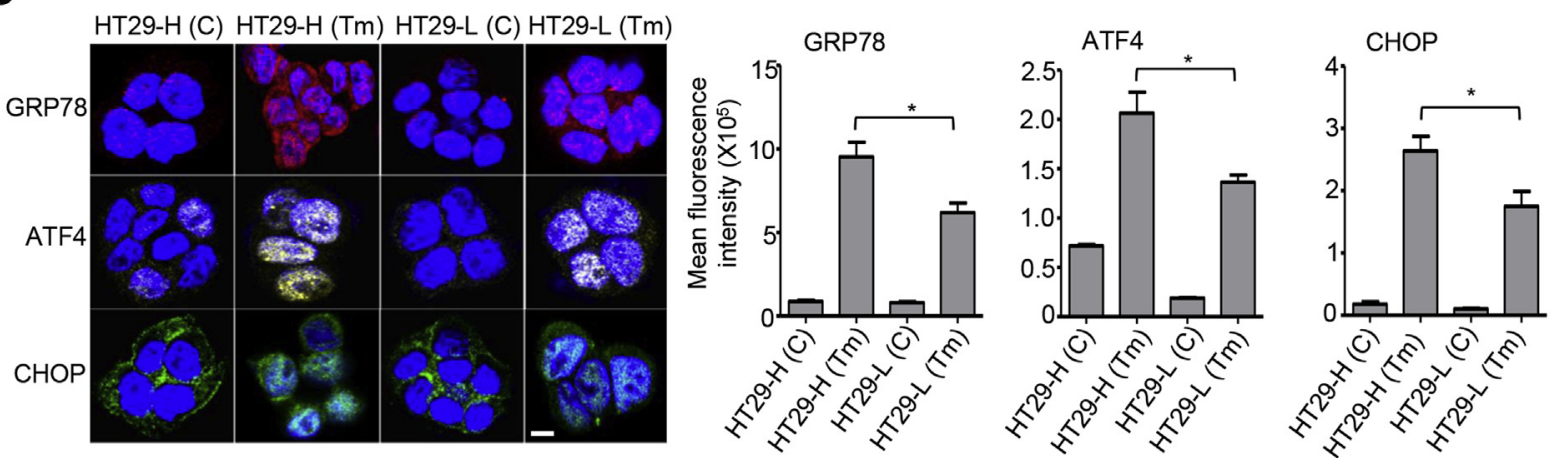

$\mathbf{E}$
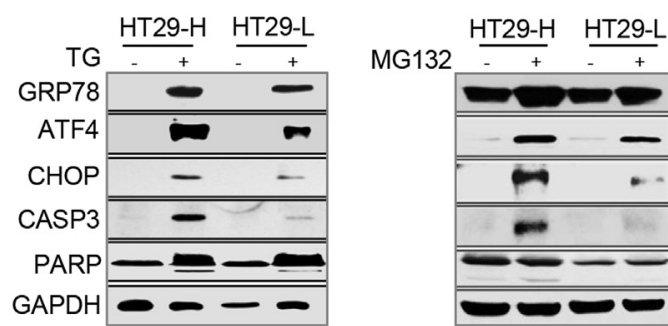

$\mathbf{F}$

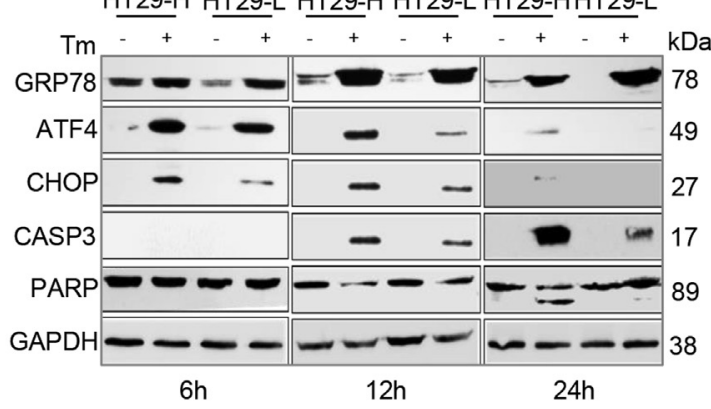

Figure $2 \mathrm{HT} 29-\mathrm{H}$ is more susceptible to endoplasmic reticulum (ER) stress and apoptosis than HT29-L cells in response to ER stress-inducing agents. A: $\mathrm{HT} 29-\mathrm{H} / \mathrm{L}$ cells were treated with $10 \mu \mathrm{g} / \mathrm{mL}$ tunicamycin $(\mathrm{Tm})$ for 6,12 , and 24 hours (h), and misfolded MUC2 and mature MUC2 were detected by Western blotting using a MUC2 non-glycosylated precursor antibody (Ab; 4F1) and mature MUC2 antibody, respectively. B-D: Cells were treated with $10 \mu \mathrm{g} / \mathrm{mL}$ Tm for 12 hours. ER stress was quantified by real-time quantitative PCR (B), at, 6, 12, and 24 hours, ER stress and apoptosis were determined by Western blotting (C), and at 12 hours, ER stress was determined by confocal microscopy (D). Fluorescence intensity was quantified from three different images. E: ER stress and apoptosis were analyzed by Western blotting after stimulation with $5 \mu \mathrm{mol} / \mathrm{L}$ thapsigargin (TG) and $10 \mu \mathrm{mol} / \mathrm{L}$ MG132 for 12 hours. F: Non-MUC2-producing Caco-2 cells exhibit less ER stress than $\mathrm{HT} 29-\mathrm{H} / \mathrm{L}$ cells. Gene expression levels were normalized using GAPDH as housekeeping gene. Data are expressed as means \pm SEM (B and D). $n=4$ (B); $n=3$ (D). ${ }^{*} P<0.05,{ }^{*} p<<0.01$, and ${ }^{*} * *<<0.001$ (t-test). Scale bar $=10 \mu \mathrm{m}$ (D). AGR, anterior gradient; ATF, activating transcription factor; $C$, control; CASP, caspase; CHOP, CCAAT-enhancer-binding protein homologous protein; GRP, glucose-regulated protein; $h$, hour; PARP, poly (ADP-ribose) polymerase; SPDEF, SAM pointed domain-containing ETS transcription factor; sXBP, spliced X-box-binding protein-1; Tm, tunicamycin.

5'-CTGCTGCCTCTAATACGCCA-3' (reverse) for mouse ATF4; 5'-AACAGAGGTCACACGCACAT-3' (forward) and 5'-TTCCTCCTCTTCCTCCTGGG-3' (reverse) for mouse CHOP; 5'-GGCCAAAGACACCACAGTCA-3' (forward) and 5'-CTGAGGTAGTTTGGGCCGAG-3' (reverse) for mouse anterior gradient $2 ; 5^{\prime}$-GACTCACACTCAAGGGGCAA-3' (forward) and 5'-TCAGAAGAGTCGTCCGTCCT-3' (reverse) for mouse SPDEF; 5'-GAAGCCAGATCCCGAA- 

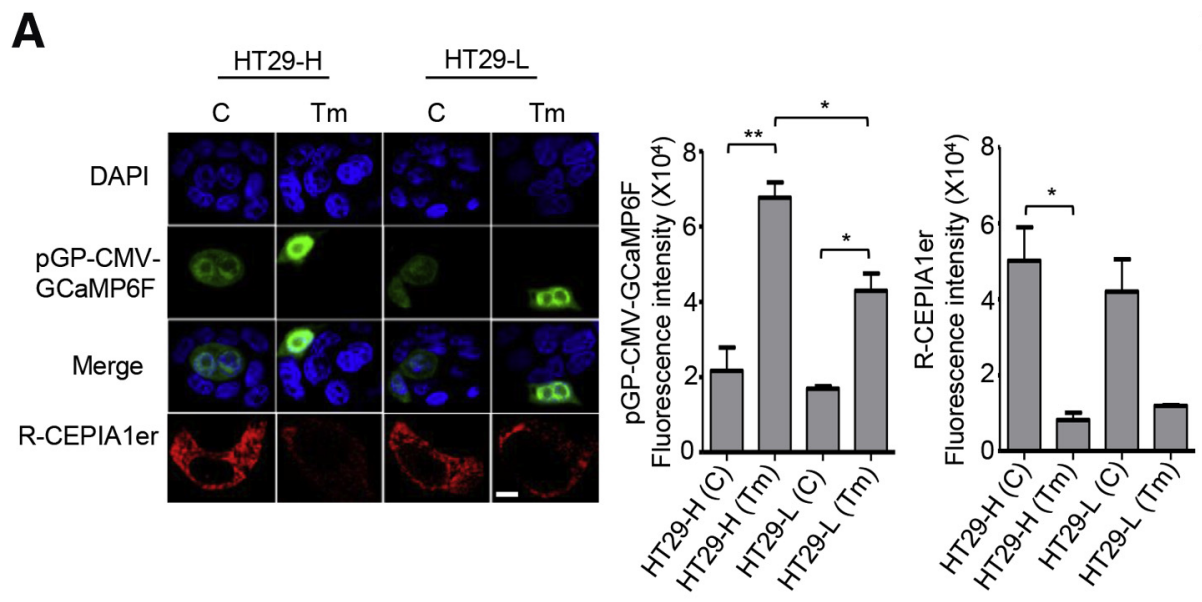

C
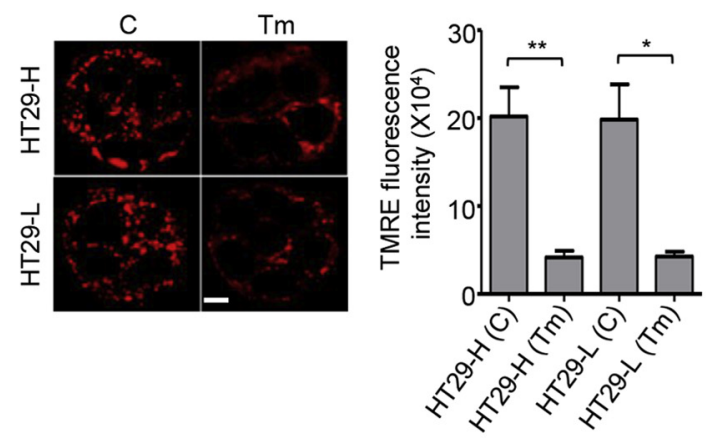

D

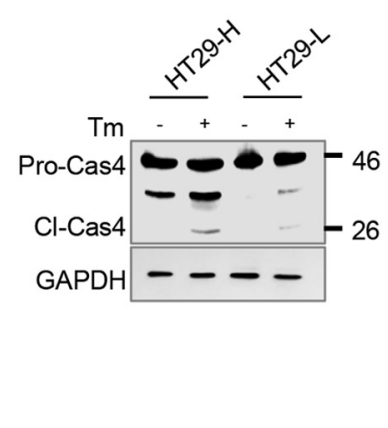

$\mathbf{F}$
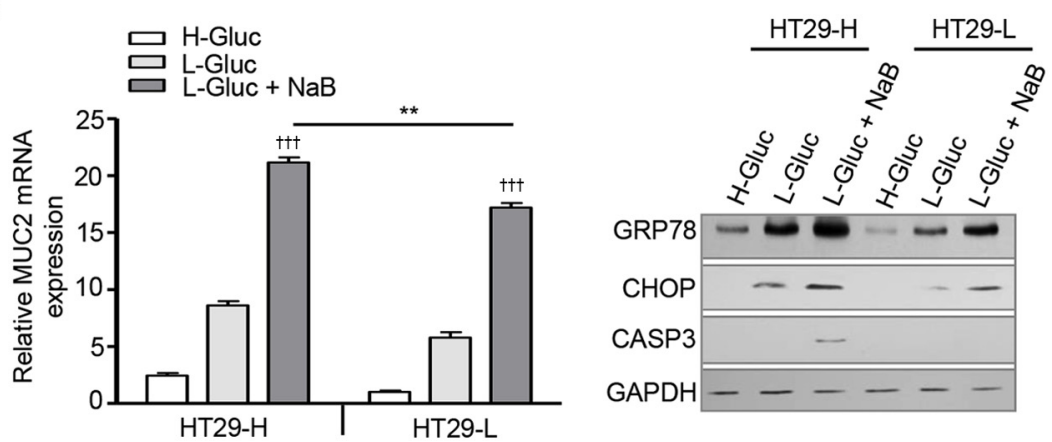

B

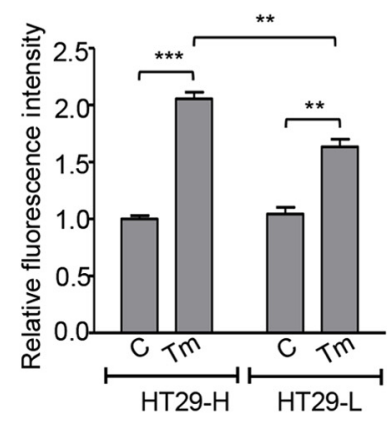

E

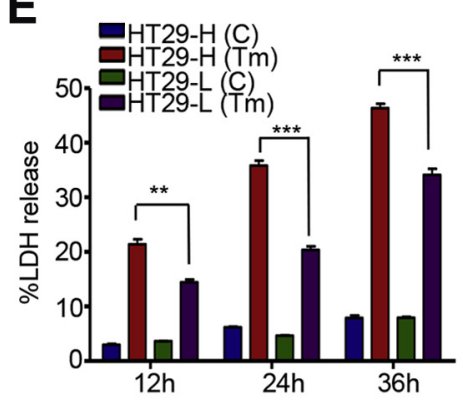

G

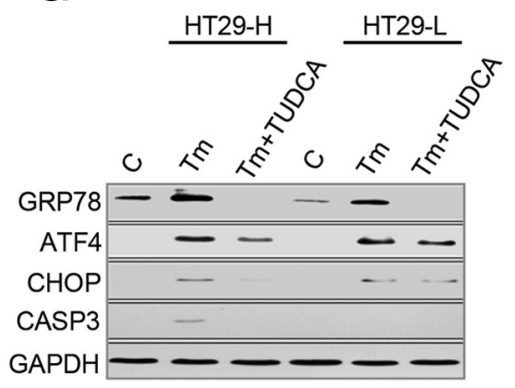

Figure 3 Endoplasmic reticulum (ER) stress stimulates various apoptosis events. A: HT29-H/L cells were transfected with fluorescent calcium sensor proteins targeted to the ER (R-CEPIA1er) and cytoplasm (pGP-CMV-GCaMP6F); changes in calcium were assessed by confocal microscopy after $10 \mu \mathrm{g} / \mathrm{mL}$ tunicamycin $(\mathrm{Tm})$ treatment, and fluorescence intensity was quantified (bar graphs). B: Cells were labeled with fluo-4 am, and intracellular calcium increase in response to Tm was analyzed with a fluorescent plate reader. C: Analysis of TMRE staining (to detect mitochondrial membrane potential) after Tm treatment by confocal microscopy, and fluorescence intensity was quantified (bar graphs). A-C: Analysis of variance with Bonferroni's post hoc test was used. D and E: HT29-H/L cells were stimulated with Tm. D: Caspase (CASP) 4 cleavage was determined by Western blot analysis. E: Lactate dehydrogenase (LDH) release was measured by spectrophotometric absorbance at $490 \mathrm{~nm}$. $t$-Test was used. F: Cells were grown in normal Dulbecco's modified Eagle's medium (DMEM; H-Gluc), low-glucose DMEM media (L-Gluc), and low-glucose DMEM containing $2 \mathrm{mmol} / \mathrm{L}$ sodium butyrate (L-Gluc $+\mathrm{NaB}$ ) for 24 hours (h), and ER stress and apoptosis were analyzed by Western blotting and real-time quantitative PCR. MUC2 mRNA expression level was normalized using GAPDH as housekeeping gene. G: Cells were pretreated for 3 hours with $1 \mathrm{mmol} / \mathrm{L}$ tauroursodeoxycholic acid (TUDCA) before stimulating with Tm, and ER stress and apoptosis were determined by Western blotting. Data are expressed as means $\pm \mathrm{SEM}(\mathbf{A}-\mathbf{C}, \mathbf{E}, \mathbf{F}) . n=3(\mathbf{A}-\mathbf{C}$ and $\mathbf{E}) .{ }^{*} P<0.05,{ }^{* *} P<0.01$, and ${ }^{* * *} P<0.001\left(t\right.$-test); ${ }^{\dagger \dagger \dagger} P<0.001$ versus $\mathrm{H}$-Gluc (analysis of variance). Scale bar $=10 \mu \mathrm{m}$ (A and C). C, control; CHOP, CCAAT-enhancer-binding protein homologous protein; Cl, cleaved; GRP, glucoseregulated protein; TMRE, tetramethylrhodamine, ethyl ester.

ACCA-3' (forward) and 5'-CCAGCTTGTGGGTGAGGTAG-3' (reverse) for mouse MUC2; and 5'-TGGGGTGTTGAAGGTCTC-3' (forward) and 5'-CTACAATGAGCTGCGTGTG- $3^{\prime}$ (reverse) for mouse $\beta$-actin. Gene expression was normalized to glyceraldehyde-3-phosphate dehydrogenase or $\beta$-actin as an endogenous control.

\section{Protein Extraction and Western Blotting}

Cells or full-thickness colonic tissues were sonicated and/or homogenized in cell lysis buffer $(1 \mathrm{mmol} / \mathrm{L}$ EDTA, $10 \mathrm{mmol} / \mathrm{L}$ Tris- $\mathrm{HCl}, 10 \%$ SDS, $5 \mathrm{mmol} / \mathrm{L} \mathrm{NaCl}, 20 \%$ Triton X-100, phenylmethylsulfonyl fluoride, and a protease 
A

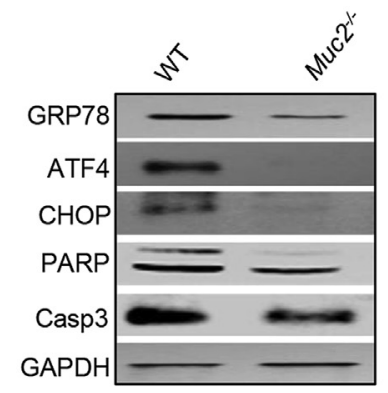

B

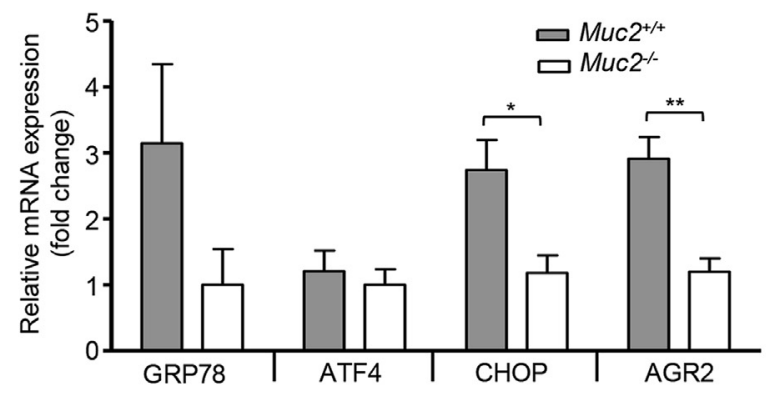

C

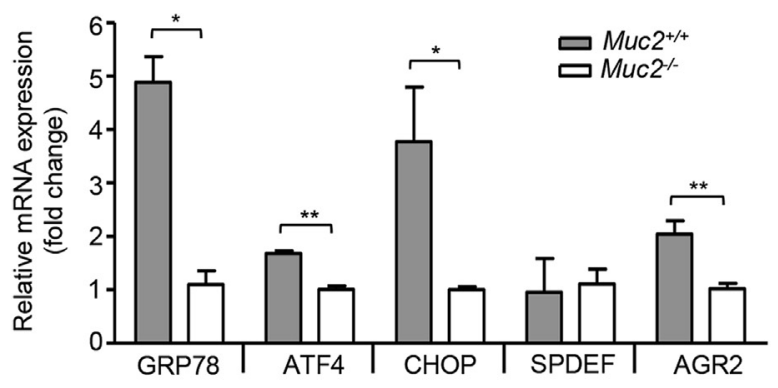

D
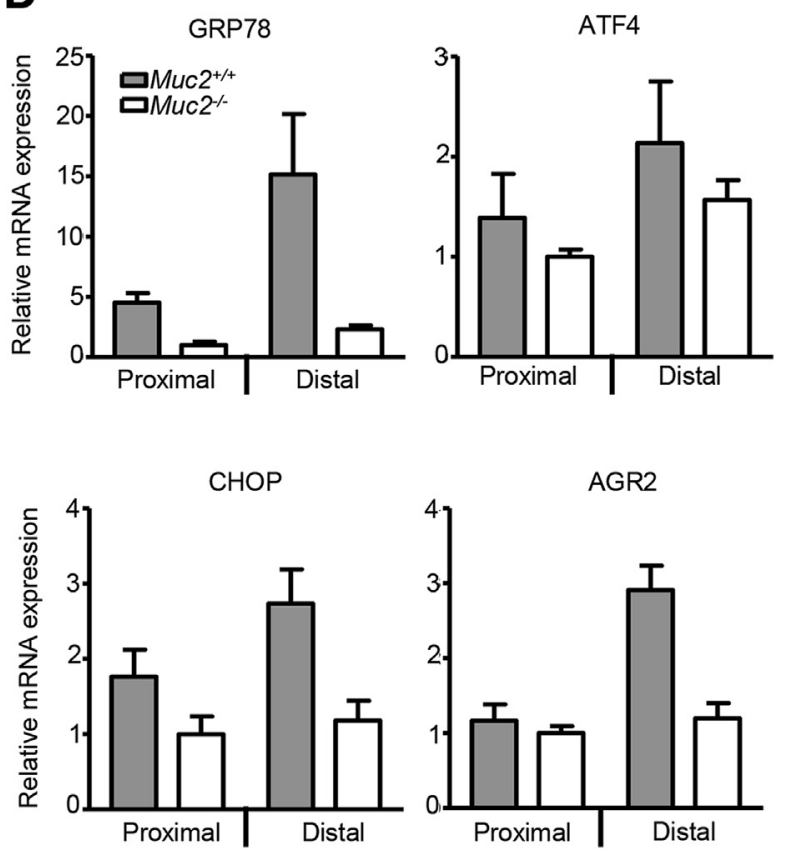

inhibitor cocktail; Sigma-Aldrich). Lysates were centrifuged at $14,000 \times g$ for 10 minutes at $4^{\circ} \mathrm{C}$, and supernatants were collected and protein content in the supernatants was determined using bicinchoninic acid reagent (Pierce; Thermo Fisher Scientific, Whitby, ON, Canada). Equal amounts of proteins were resolved by electrophoresis on $12 \%$ or $7.5 \%$ SDS polyacrylamide gels and transferred onto nitrocellulose membranes. Blots were blocked with $5 \%$ skim milk in PBS containing $0.1 \%$ Tween 20 for 1 hour and incubated overnight at $4{ }^{\circ} \mathrm{C}$ with primary antibodies, against GRP78 (3177; Cell Signaling, Danvers, MA), ATF4 (11,815; Cell Signaling), CHOP (2895; Cell Signaling), cleaved caspase 3 (9661; Cell Signaling), poly (ADP-ribose) polymerase (9542; Cell Signaling), and glyceraldehyde-3phosphate dehydrogenase (569397; Calbiochem). The blots were washed with PBS containing $0.1 \%$ Tween 20, incubated with appropriate secondary antibodies for 1 hour at room temperature, and developed using electrochemiluminescence Western blotting detection reagent (GE Healthcare, Mississauga, ON, Canada).

\section{Immunofluorescence Staining and Confocal Microscopy}

For immunological staining, HT29-H/L cells were grown on coverslips, and after treatment, immunocytochemical procedures were performed to detect GRP78, ATF4, CHOP, and DNA fragmentation. Cells were fixed with $3.5 \%$ paraformaldehyde for 15 minutes at room temperature and permeabilized with $0.35 \%$ Triton X-100 for 5 minutes at room temperature. The cells were then blocked with $2 \%$ donkey serum in PBS containing $0.1 \%$ Tween 20 for 1 hour and incubated with primary antibodies overnight. Mouse tissues were also stained with tetrarhodamine isothiocyanate-conjugated Ulex europaeus agglutinin 1 (L4889; Sigma), anti-MUC2 antibody (Sc-15334; Santa Cruz Biotechnology, Dallas, TX), cleaved caspase 3, and CHOP. After washing, cells/tissues were incubated for 2 hours with appropriate secondary antibodies. GRP78 and ATF4 were detected with anti-rabbit Alexa Fluor 594, and CHOP was detected with anti-mouse Alexa Fluor 488.

Figure 4 Endoplasmic reticulum (ER) stress is higher in $\mathrm{MuC2}^{+/+}$than Muc2 $^{-/-}$littermates. A and B: Full-thickness colon tissues from Muc2 ${ }^{+/+}$ and $\mathrm{MuC2}^{-/-}$littermates were isolated and analyzed for ER stress and apoptosis by Western blot analysis (A) and real-time quantitative PCR (qPCR) (B). C: Isolated colonic epithelial cells from Muc2 $2^{+/+}$and $\mathrm{MuC2}^{-/-}$ mice were analyzed for markers of ER stress by qPCR. D: Full-thickness colon tissues from the proximal and distal colon of $\mathrm{MuC2}^{+/+}$and $\mathrm{MuC2}^{-/-}$littermates were isolated and analyzed for ER stress markers by qPCR. Gene expression levels were normalized using $\beta$-actin as housekeeping gene. Data are expressed as means \pm SEM (B-D). $n=5$ mice (B); $n=8$ mice (C and D). ${ }^{*} P<0.05,{ }^{*} P<<0.01$ (t-test). AGR, anterior gradient; ATF, activating transcription factor; Casp, caspase; CHOP, CCAAT-enhancerbinding protein homologous protein; GAPDH, glyceraldehyde-3-phosphate dehydrogenase; GRP, glucose-regulated protein; PARP, poly (ADP-ribose) polymerase; SPDEF, SAM pointed domain containing ETS transcription factor; WT, wild type. 
Nuclei were counterstained with DAPI, and cells were mounted with fluorSave reagent (Calbiochem; EMD Millipore, Billerica, MA). The slides were examined using FluoView FV1000 confocal immunofluorescence microscope (Olympus, Tokyo, Japan). Fluorescence intensity of images was quantified using FIJI (ImageJ version 1.45s; NIH, Bethesda, MD; http://imagej.nih.gov/ij).

\section{Measurement of Reactive 0xygen Species}

HT29-H/L cells were plated in a 96-well plate and stained with $25 \mu \mathrm{mol} / \mathrm{L} \quad 2^{\prime}, 7^{\prime}$-dichlorofluorescin diacetate (ab113851; Abcam, Toronto, ON, Canada) for 45 minutes at $37^{\circ} \mathrm{C}$. The cells were then treated with tunicamycin. Fluorescent signal was read at excitation of $485 \mathrm{~nm} / \mathrm{emission}$ of $535 \mathrm{~nm}$ with a fluorescent microplate reader.

\section{Cell Viability Assay}

The cells were seeded in a 96-well plate to approximately $80 \%$ confluence, MTT assay was performed after the required treatments (Sigma-Aldrich), and $0.5 \mathrm{mg} / \mathrm{mL}$ MTT was added to each well and incubated for 4 hours. The medium was removed, and the cells were washed with PBS and solubilized in dimethyl sulfoxide (Sigma-Aldrich). Absorbance was read at $595 \mathrm{~nm}$.

\section{Killing Assay with Entamoeba histolytica}

HT29-H/L cells were cultured in 12-well plates and treated with $E$. histolytica. The live virulent axenic $E$. histolytica strain HM1:IMSS was harvested in the logarithmic phase of growth by chilling the cultures on ice for 10 minutes to detach the cells. The cells were then centrifuged at $200 \times g$ for 5 minutes at $4{ }^{\circ} \mathrm{C}$, resuspended in M199 medium supplemented with $5.7 \mathrm{mmol} / \mathrm{L}$ cysteine, $25 \mathrm{mmol} / \mathrm{L}$ HEPES, and $0.5 \%$ bovine serum albumin, and adjusted to $1 \times 10^{5}$ amoebas/mL. M199 (1 mL) was added to control wells (no amoebas), and media-containing amoeba was added to sample wells. The plates were incubated for 3 hours at $37^{\circ} \mathrm{C}$. After incubation, plates were left on ice for $30 \mathrm{mi}-$ nutes to detach amoebas and then washed off with ice-cold PBS. The cell monolayer was fixed with $2.5 \%$ glutaraldehyde for 10 minutes at room temperature and stained with $0.1 \%$ methylene blue for 10 minutes at room temperature. $\mathrm{HCl}(1 \mathrm{~N})$ was added to each well, and plates were incubated for 30 minutes at $37^{\circ} \mathrm{C}$ to extract the stain. After incubation, the supernatants were collected and absorbance was read at $660 \mathrm{~nm}$. Percentage monolayer destruction was calculated as follows: $\left[\mathrm{OD}_{660}\right.$ (control wells) $-\mathrm{OD}_{660}$ (experimental wells $)] /\left[\mathrm{OD}_{660}\right.$ (control wells) $\left.\times 100\right]$.

\section{Mice}

Wild-type C57BL/6 $\mathrm{Muc}^{+/+}$and $\mathrm{Muc}^{-/-}$mice on the same genetic background were used in experiments. Wild- type mice were purchased from Charles River Laboratories International (St-Constant, QC, Canada), and $\mathrm{Muc}^{-1-}$ mice obtained from Dr. Anna Velcich (Albert Einstein College of Medicine/Albert Einstein Cancer Center, New York, NY) were bred in house to generate littermate $\mathrm{Muc}^{+/-}$breeding pairs. Math1 ${ }^{\text {M1GFP }}$ (strain 013593) mice on a C57BL/6 background were purchased from The Jackson Laboratory (Bar Harbor, ME). All mice used were 10 to 12 weeks old. The mice were kept in sterilized filter-top cages and maintained on standard food and water ad libitum. The Animal Ethics Committee of the University of Calgary approved all studies.

\section{Induction of Colitis}

To assess ER stress and apoptosis of goblet cells in C57BL/ $6 \mathrm{Muc}^{+/+}$mice during early onset of colitis, mice were randomly divided into two groups: a control group that received only water and a colitis group that received 3.5\% $(\mathrm{w} / \mathrm{v})$ dextran sulfate sodium (DSS) in drinking water. The animals were sacrificed daily, and colonic tissue samples were fixed in Carnoy's solution or $4 \%$ paraformaldehyde for immunohistochemical analysis. RNA was also extracted with TRIzol reagent, purified of DSS before performing real-time quantitative PCR analysis. To purify DSS, the extracted RNA was incubated with $2 \mathrm{~mol} / \mathrm{L}$ lithium chloride for 2 hours on ice. It was then centrifuged at $14,000 \times g$ for 30 minutes at $4^{\circ} \mathrm{C}$. The supernatant was removed, and the process was repeated. RNA was then precipitated with $3 \mathrm{~mol} / \mathrm{L}$ sodium acetate and $100 \%$ absolute ethanol, washed with $70 \%$ ethanol, and dissolved in RNAase-free water.

\section{Isolation of Colonic Epithelial Cells from Mice}

Colonic epithelial cells from C57BL/6 $\mathrm{Muc}^{+/+}$and $M u c 2^{-1-}$ mice were isolated by flushing colon tissues twice with PBS to remove feces, after which they were washed once with $1 \mathrm{mmol} / \mathrm{L}$ dithiothreitol in PBS. Colons were inverted, cut into 3-mm pieces, and incubated at $37^{\circ} \mathrm{C}$ under agitation for 30 minutes in PBS containing $200 \mathrm{U} / \mathrm{mL}$ collagenase (C6885; Sigma-Aldrich) and 100 U/mL hyaluronidase (H3506; Sigma-Aldrich). Incubation solutions were removed, and the cells were sieved through a cell strainer $(400 \mu \mathrm{m})$. The flow-through was centrifuged at $300 \times g$ for 5 minutes at $4{ }^{\circ} \mathrm{C}$, and the resulting pellet was washed twice with ice-cold PBS. For gene expression analysis, RNA was extracted using TRIzol reagent. For flow cytometric analysis, colonic epithelial cells from Math1 ${ }^{\text {MiGFP }}$ mice were isolated by collagenase hyaluronidase digestion, as described earlier in this section, and washed with fluorescence-activated cell sorting buffer containing calcium magnesium-free PBS, $2 \mathrm{~mm}$ EDTA, and $1 \%$ fetal calf serum. Cells were then stained with $0.06 \mu \mathrm{g} / 10^{6}$ cells of CD326eFluor780 (eBioscience: 47-5791; Thermo Fisher) and CD45-eFluor450 (eBioscience: 48-0451). 
A
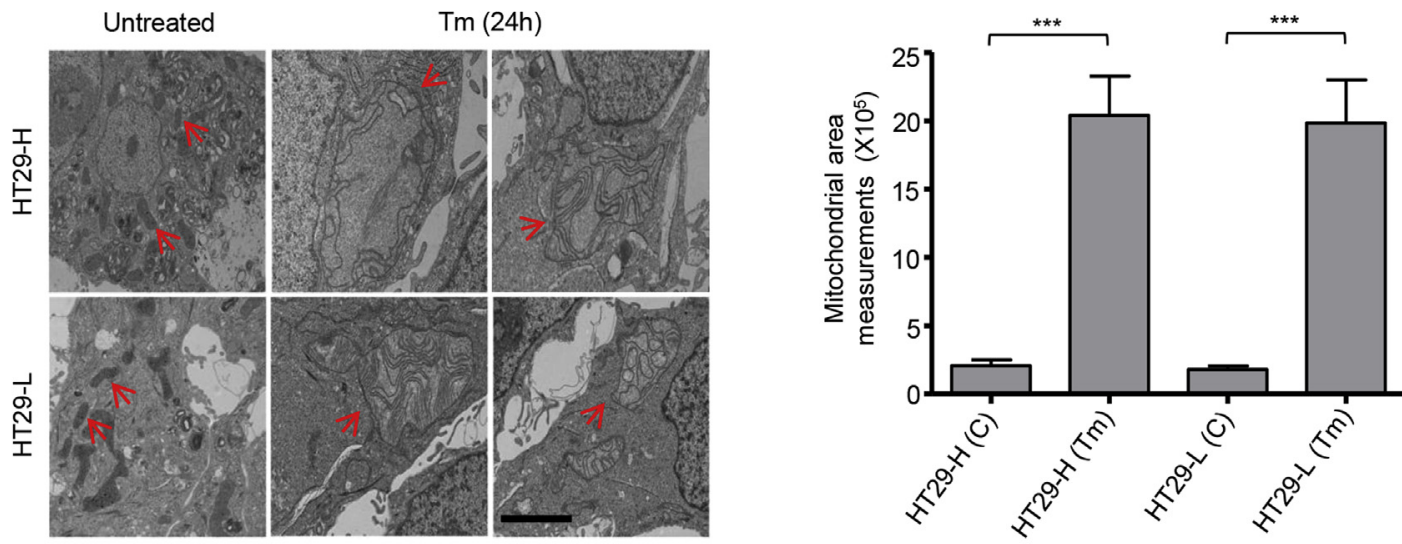

B

C

D
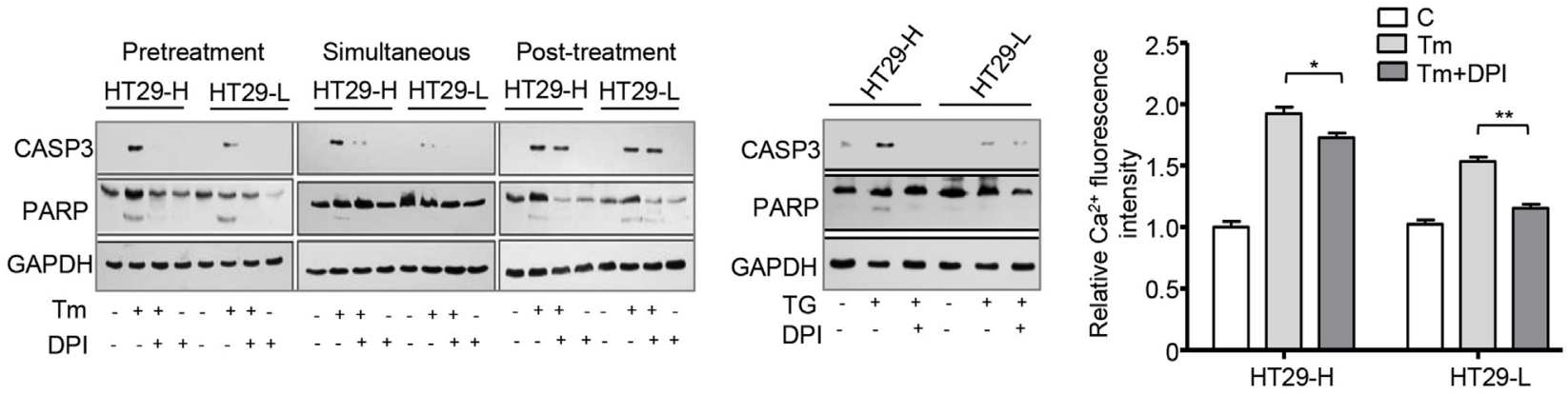

E

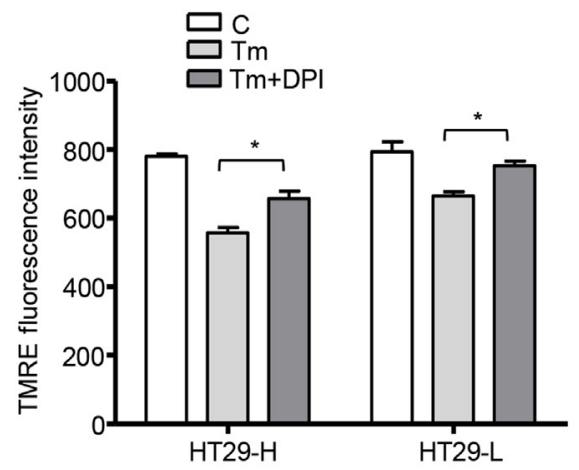

$\mathbf{F}$
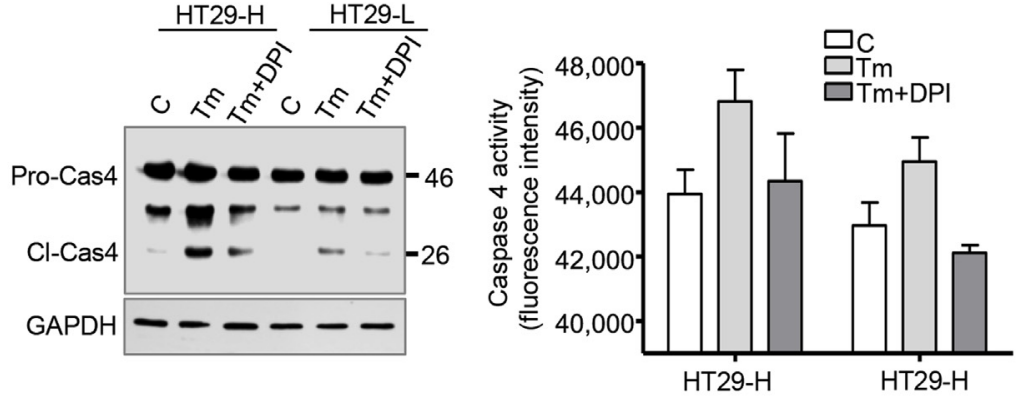

H

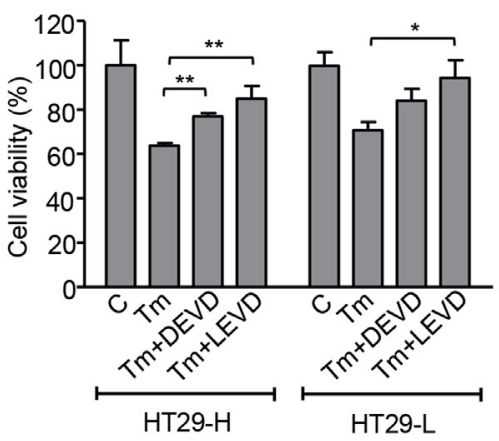




\section{Statistical Analysis}

All statistical analyses were performed using GraphPad Prism version 5.01 (GraphPad Software, San Diego, CA). $t$-Test and analysis of variance with post hoc tests were used to compare groups. Significant differences between treatment groups were assessed at $P<0.05$. Error bars denote SEM.

\section{Results}

High MUC2 Mucin Production Increases Susceptibility of Goblet Cells to Apoptosis

The role of high MUC2 production was investigated in susceptibility of goblet cells to apoptosis, using two goblet cell-like cell lines: a high MUC2-producing cell (HT29$\mathrm{H})^{23}$ and an HT29-H clone (HT29-L) that produces and secretes significantly less ${ }^{3} \mathrm{H}$-glucosamine-labelled MUC2 (Figure 1, A-D). In response to staurosporine, apoptosis [cleaved poly (ADP-ribose) polymerase, caspase 3 activation, and DNA fragmentation] and cell death [lactate dehydrogenase release] was significantly higher in HT29-H than HT29-L cells (Figure 1, E-G). To determine whether other apoptosis-inducing agents may induce a similar response, cells were stimulated with a combination of interferon- $\gamma$ and tumor necrosis factor- $\alpha$, known to cause intestinal epithelial cell apoptosis, to mimic proinflammatory conditions in the gut. ${ }^{24}$ Like staurosporine, HT29-H cells were substantially more susceptible to apoptosis than HT29-L cells. Apoptosis only occurred in HT29-L cells with a high dose $(20 / 80 \mathrm{nmol} /$ L) of interferon- $\gamma /$ tumor necrosis factor- $\alpha$ (Figure $1 \mathrm{H}$ ). Lactate dehydrogenase release was also significantly higher in HT29-H than HT29-L cells in a temporal manner (Figure 1I). To verify if high MUC2 biosynthesis was driving the increase in susceptibility to apoptosis, the sensitivity of Caco-2 colonic epithelial cells, which do not produce MUC2, was compared with that of HT29-H/L cells, and they were found to be resistant to apoptosis. Caspase 3 and poly (ADP-ribose) polymerase cleavages were not detected, whereas HT29-H/L cells showed considerable apoptosis (Figure 1J). These data show that increased MUC2 output rendered goblet cells sensitive to death in the presence of apoptosis-inducing agents.

\section{High MUC2 Biosynthesis Increases Goblet Cell Susceptibility to ER Stress}

ER stress results from accumulation of unfolded/misfolded proteins in the ER, and goblet cells are prone to this condition because of the large amounts of MUC2 (likely to misfold because of its large size) they produce. ${ }^{25}$ To determine whether ER stress played a role in MUC2induced susceptibility to apoptosis, the expression of ER stress markers was analyzed in cells under basal conditions and when treated with ER stress-inducing agents. Under basal conditions, MUC2 precursor was detected in HT29-H/L cells as well as mature MUC2 in HT29-H cells (Figure 2A). As predicted, inhibiting N-linked glycosylation with tunicamycin markedly induced misfolding. Non-glycosylated MUC2 was evident by a shift on the Western blot that migrated at a different rate, with a corresponding decrease in mature MUC2, consistent with increased MUC2 misfolding (Figure 2A). Not surprising, other goblet cell peptides, such as trefoil factor 3 and resistin-like molecule- $\beta$, were also reduced with tunicamycin treatment (Supplemental Figure S2). Under these conditions, ER stress markers were significantly increased at the mRNA and protein level in HT29-H versus HT29-L cells (Figure 2, B-D). HT29-H cells also exhibited significantly higher ER stress than HT29-L cells in response to the ER stressors thapsigargin and MG132 (Figure 2E). Predictably, non-MUC2producing Caco-2 cells exhibited less ER stress than HT29-H/L cells (Figure 2F). During prolonged ER stress (12 and 24 hours), HT29-H cells had a higher rate of apoptosis than HT29-L cells. Under these conditions, Caco2 cells were resistant to ER stress-induced apoptosis (Figure 2F). Other apoptosis events activated during ER stress included increase in cytoplasmic calcium, depletion of ER calcium (Figure 3, A and B), and loss of mitochondrial membrane potential (Figure 3C). Loss of mitochondrial membrane potential was similar in both HT29-H/L cells, but increase in cytoplasmic calcium was significantly higher in HT29-H than HT29-L cells. Interestingly, caspase 4, which plays a role in ER stress-induced apoptosis, was more activated in HT29-H than HT29-L cells (Figure 3D). Cell death under ER stress conditions was significantly higher in HT29-H than HT29-L cells (Figure 3E).

\footnotetext{
Figure 5 Endoplasmic reticulum (ER) stress-induced apoptosis is dependent on reactive oxygen species. A: $\mathrm{HT} 29-\mathrm{H} / \mathrm{L}$ cells were treated with $10 \mu \mathrm{g} / \mathrm{mL}$ tunicamycin (Tm) for 12 hours and analyzed by transmission electron microscopy, and area of mitochondria was measured. Arrows show normal mitochondria (untreated cells) and enlarged mitochondria (tunicamycin-treated cells). $t$-Test was used. B and C: Cells were exposed to $10 \mu$ mol/L diphenyleneiodonium (DPI) before, during, or after Tm treatment ( $10 \mu \mathrm{mol} / \mathrm{L}$ for 12 hours; B) and before thapsigargin (TG) treatment (5 $\mu \mathrm{mol} / \mathrm{L}$ for 12 hours) (C), and apoptosis was determined by Western blotting. D-F: Cells were pretreated with DPI before Tm treatment, and intracellular calcium levels (D), mitochondrial membrane potential (E), and caspase (CASP) 4 cleavages and activity (F) were measured. G and H: HT29-H/L cells were pretreated with $50 \mu \mathrm{mol} / \mathrm{L}$ Z-LEVD-FMK [Z-Leu-Glu(OMe)-Val-Asp(OMe)-fluoromethylketone] or Z-DEVD-FMK [Z-Asp(OMe)-Glu(OMe)-Val-Asp(OMe))-fluoromethylketone] DPI before $10 \mu \mathrm{g} / \mathrm{mL}$ tunicamycin treatment for 12 hours, apoptosis was analyzed by terminal deoxynucleotidyl transferase-mediated dUTP nick-end labeling (TUNEL) assay (G), and cell viability was analyzed by MTT assay (H). D, E, and $\mathbf{H}$ : Analysis of variance with Bonferroni's post hoc test was used. Data are expressed as means \pm SEM $(\mathbf{A}, \mathbf{D}, \mathbf{E}, \mathbf{F}$, and $\mathbf{H}) . n=3(\mathbf{D}, \mathbf{E}$, and $\mathbf{H}) .{ }^{*} P<0.05,{ }^{* *} P<0.01$, and ${ }^{* * *} P<0.001$. Scale bars: $500 \mathrm{~nm}(\mathbf{A}) ; 10 \mu \mathrm{m}(\mathbf{G})$. C, control; Cl, cleaved; DEVD, Z-Asp(OMe)-Glu(OMe)-Val-Asp(OMe)-fluoromethylketone; GAPDH, glyceraldehyde-3-phosphate dehydrogenase; PARP, poly (ADP-ribose) polymerase; TMRE, tetramethylrhodamine, ethyl ester.
} 
A
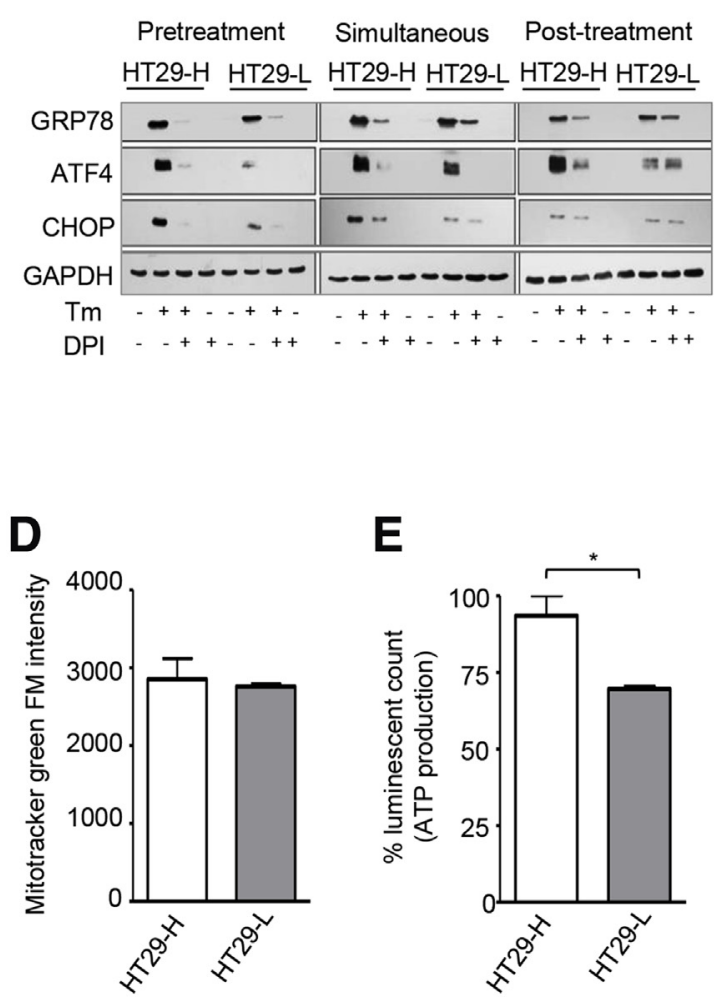

B

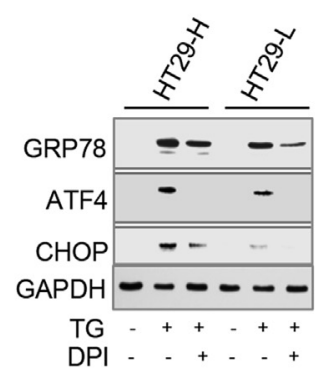

C

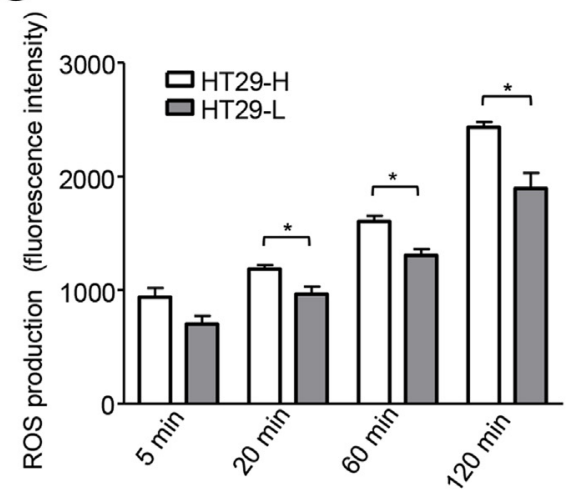

$\mathbf{F}$

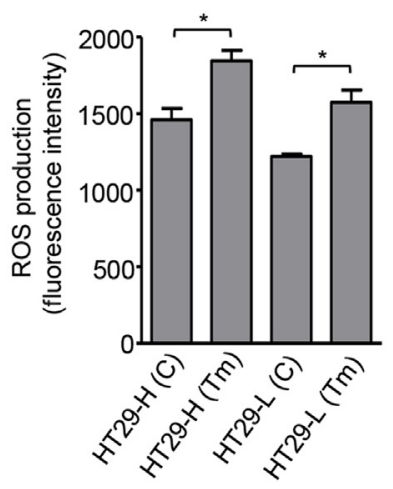

G

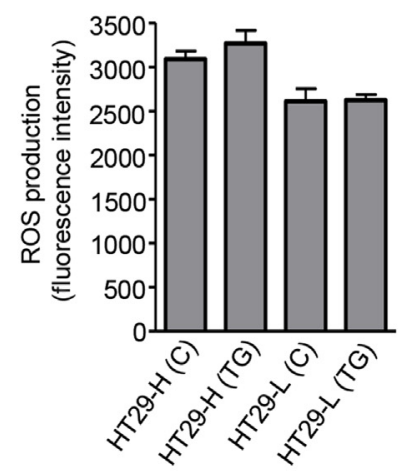

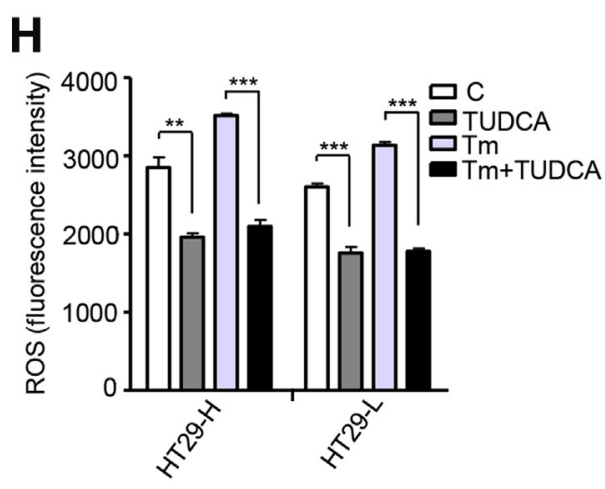
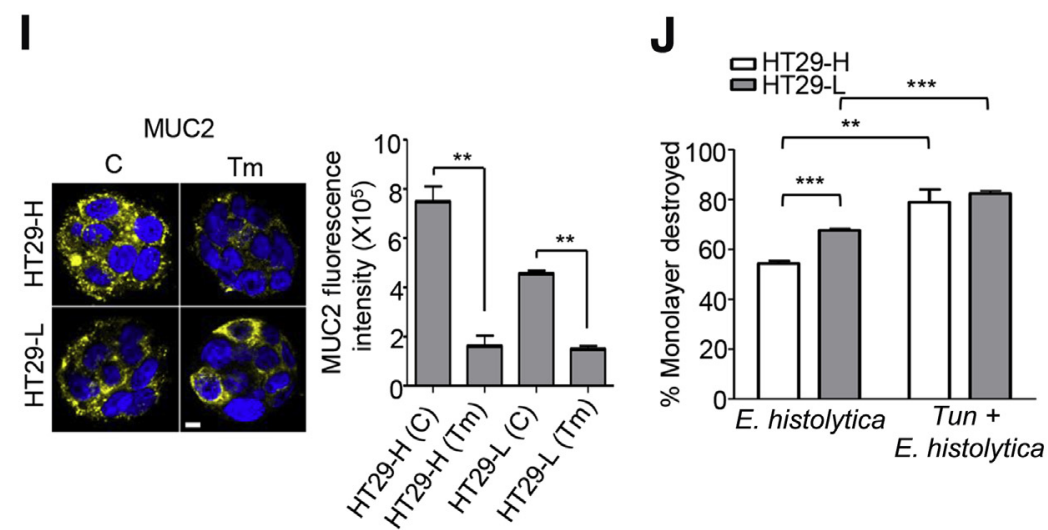

Figure 6 Endoplasmic reticulum (ER) stress is dependent on reactive oxygen species (ROS). A and B: Cells were exposed to $10 \mu \mathrm{mol} / \mathrm{L}$ diphenyleneiodonium (DPI) before, during, or after tunicamycin (Tm) treatment (A) and before thapsigargin (TG) treatment (B), and ER stress was determined by Western blotting. C: Basal ROS production was measured by staining with dichlorofluorescein diacetate dye, and ROS were quantified using a fluorescent plate reader. D: Cells were stained with mitotracker green, and mitochondrial mass was quantified using a fluorescent plate reader. E: Basal ATP production in HT29-H/L cells. F and G: Cells were treated with $\mathrm{Tm}(\mathbf{F})$ or TG (G), and ROS were quantified using a fluorescent plate reader. H: Cells were pretreated with tauroursodeoxycholic acid (TUDCA) for 1 hour before stimulating with Tm for 1 hour. ROS were measured with a fluorescent plate reader. I: Cells were treated with $10 \mu \mathrm{g} / \mathrm{mL} \mathrm{Tm}$ for 12 hours, MUC2 production was imaged using confocal microscopy, and fluorescence intensity was quantified. C $\mathbf{G}$ and I: $t$-Test was used. J: Cells were challenged with Entamoeba histolytica without Tm treatment or after pretreatment with $10 \mu \mathrm{g} / \mathrm{mL} \mathrm{Tm}$ (12 hours). The percentage monolayer destroyed was determined using a standard amoeba-killing assay. ${ }^{28} \mathbf{H}$ and $\mathbf{J}$ : Analysis of variance with Bonferroni's post hoc test was used. Data are expressed as means \pm SEM $(\mathbf{C}-\mathbf{J}) . n=3(\mathbf{C}-\mathbf{J}) .{ }^{*} P<0.05$, ${ }^{* *} P<0.01$, and ${ }^{* *} P<0.001$. Scale bar $=10 \mu \mathrm{m}(\mathrm{I})$. ATF, activating transcription factor; C, control; CHOP, CCAAT-enhancer-binding protein homologous protein; GAPDH, glyceraldehyde-3-phosphate dehydrogenase; GRP, glucose-regulated protein; min, minute.

To further show that high MUC2 biosynthesis increased susceptibility to ER stress and apoptosis, HT29-H/L cells were cultured in the presence of sodium butyrate (which has been shown to increase MUC2 expression considerably). ${ }^{26}$
Sodium butyrate significantly increased MUC2 expression in the cells, leading to increased ER stress and apoptosis (Figure 3F). Inhibiting ER stress with the chemical chaperone TUDCA alleviated apoptosis, suggesting specificity 


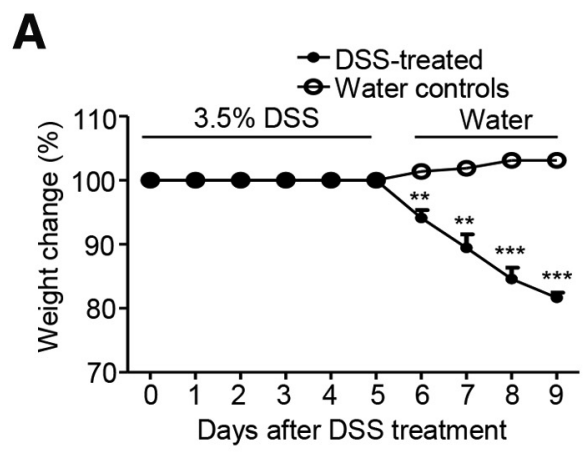

B
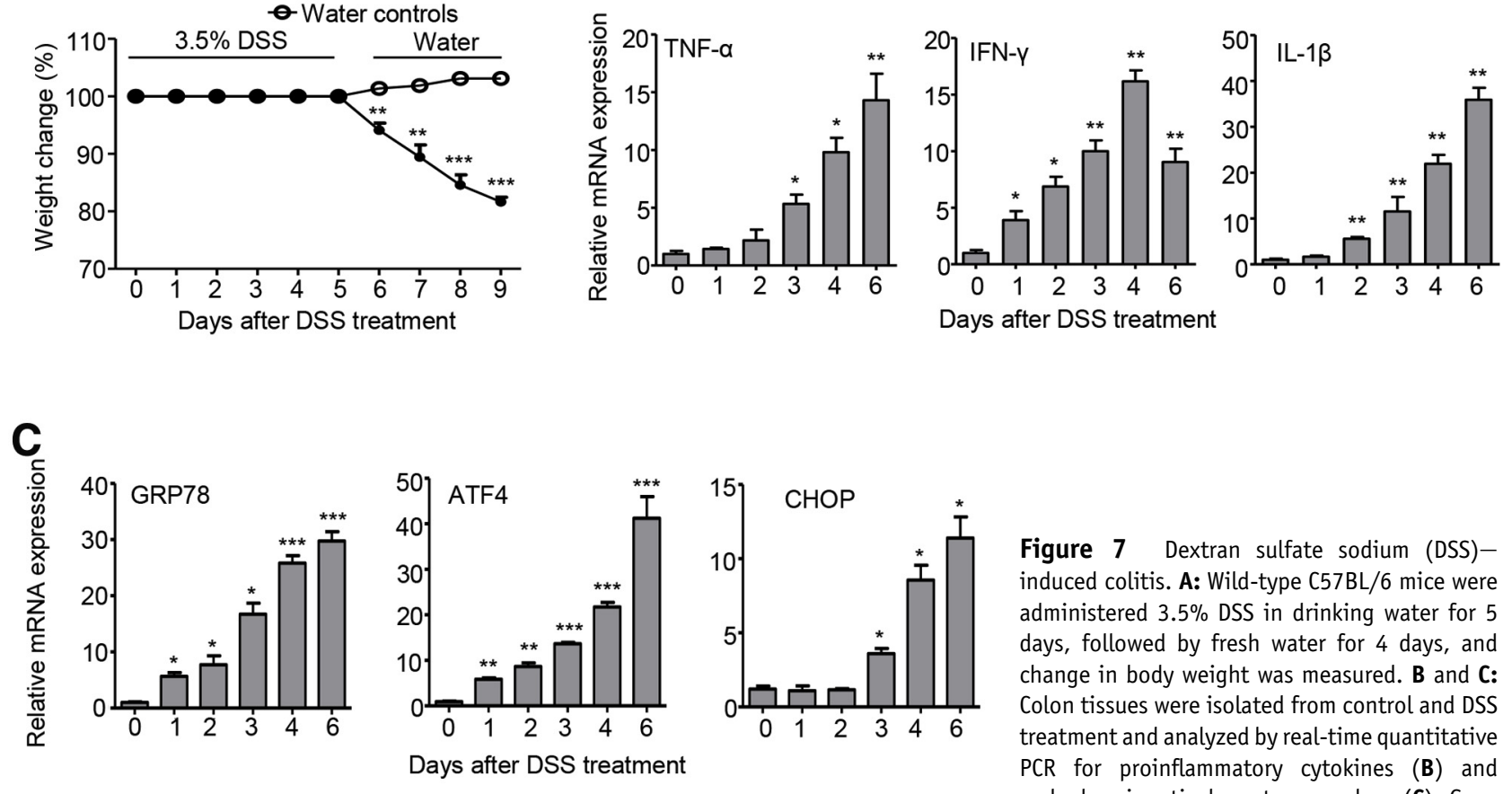

D

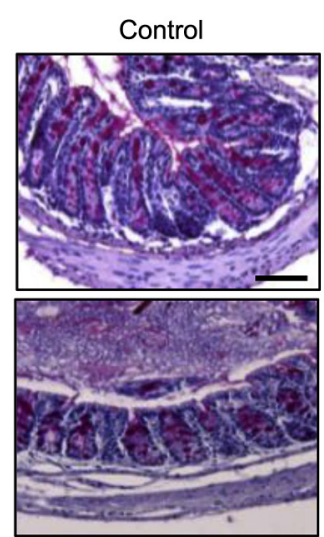

Day 3
Day 1

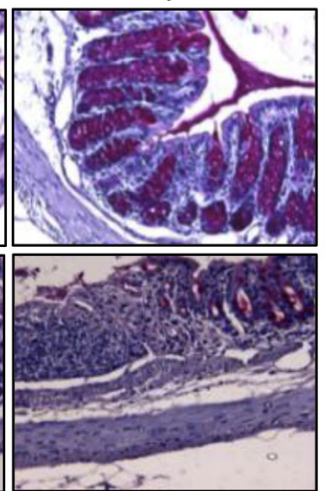

Day 4

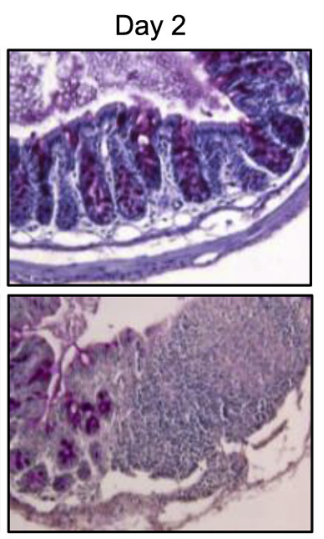

Day 6
Figure 7 Dextran sulfate sodium (DSS)induced colitis. A: Wild-type C57BL/6 mice were administered $3.5 \%$ DSS in drinking water for 5 days, followed by fresh water for 4 days, and change in body weight was measured. B and C: Colon tissues were isolated from control and DSS treatment and analyzed by real-time quantitative PCR for proinflammatory cytokines (B) and endoplasmic reticulum stress markers $(\mathbf{C})$. Gene expression levels were normalized using $\beta$-actin as housekeeping gene. D: Paraffin-embedded colon tissues ( $7 \mu \mathrm{m}$ thick) were stained with periodic acid-Schiff reagent to visualize magentastaining mucin granules in goblet cells and secreted mucus in the lumen. Data are expressed as means \pm SEM $(\mathbf{A}-\mathbf{C}) \cdot n=5$ animals per day (A); $n=4$ mice (B and C). ${ }^{*} P<0.05,{ }^{*} P<0.01$, and ${ }^{* *} P<0.001$ versus day 0 (analysis of variance with Bonferroni's post hoc test). Scale bar $=50 \mu \mathrm{m}$ (D). ATF, activating transcription factor; CHOP, CCAAT-enhancer-binding protein homologous protein; GRP, glucose-regulated protein; IFN- $\gamma$, interferon- $\gamma$; TNF- $\alpha$, tumor necrosis factor- $\alpha$. of ER stress in high MUC2-producing HT29-H cells (Figure 3G). These data demonstrate that high MUC2 production increases MUC2 misfolding and causes goblet cells to be susceptible to ER stress and subsequent apoptosis.

In vivo, full-thickness colonic tissues and isolated colonic epithelial cells revealed substantially higher ER stress and apoptosis in $\mathrm{Muc}^{+/+}$than $\mathrm{Muc} 2^{-/-}$littermates (Figure 4, $\mathrm{A}-\mathrm{C})$. In particular, the ER disulfide isomerase anterior gradient 2, important in mucin biosynthesis, was highly expressed in $M u c 2^{+/+}$versus $M u c 2^{-/-}$mice because anterior gradient 2 is up-regulated by the unfolded protein response. $^{27}$ Interestingly, isolated epithelial cells from $M u c 2^{+/+}$mice showed a slightly higher, but nonsignificant, ER stress in the distal colon (Figure 4D). These results show that Muc2 production in $\mathrm{Muc}^{+/+}$animals increases ER stress and apoptosis in the colon and suggests that goblet cells, rather than other enterocytes, are more sensitive to apoptosis in the presence of ER stressors in the gut.

\section{ER Stress-Induced Apoptosis Is Dependent on ROS}

To determine the mechanism of ER stress-induced apoptosis, it was first determined if there was any damage to the mitochondria under conditions of ER stress. ER stress is tightly linked to mitochondrial dysfunction and reactive oxygen species (ROS) production. ${ }^{28}$ By transmission electron microscopy, tunicamycin-treated HT29-H/L cells showed significantly enlarged mitochondria that were distorted (Figure 5A). Because mitochondria damage can cause increased ROS production, ${ }^{29}$ it was determined if ROS played a 
role in increasing apoptosis. Cells were pretreated, simultaneously treated, or post-treated with the ROS inhibitor DPI, and tunicamycin-induced apoptosis was determined. Pretreatment and simultaneous treatments were most effective in reducing apoptosis, whereas post-treatment only slightly reduced apoptosis (Figure 5B). Likewise, pretreatment with DPI alleviated thapsigargin-induced apoptosis (Figure 5C). DPI also significantly reduced intracellular calcium (Figure 5D), partially restored mitochondrial membrane potential (Figure 5E), and significantly attenuated caspase 4 cleavage and activity (Figure 5F). These data show that ROS played an important role in regulating ER stress-induced apoptosis. Because both caspases 3 and 4 were activated during ER stress and were markedly inhibited with DPI, it was determined if inhibiting these caspases could rescue the cells from apoptosis. Murine caspase 12 shares high homology with human caspase $4^{30}$ and acts as an initiator caspase; therefore, caspase 4 may act in a similar manner. ${ }^{31}$ Cells were pretreated with Z-LEVDFMK, a caspase 4 inhibitor, to determine whether caspase 4 was upstream of caspase 3 , but caspase 3 cleavage was not inhibited. However, pretreatment with Z-LEVD-FMK alleviated apoptosis, even to a slightly greater extent than Z-DEVDFMK (a specific caspase 3 inhibitor), suggesting that caspase 4 was as important in affecting apoptosis as caspase 3 (Figure 5G). Furthermore, pretreatment with Z-DEVD-FMK and Z-LEVD-FMK before tunicamycin stimulation partially restored cell viability by $13 \%$ and $22 \%$, respectively (Figure $5 \mathrm{H}$ ). These data demonstrate that caspases 3 and 4 are both important as effector caspases in ER stress-induced apoptosis, and inhibiting these caspases could rescue cells from apoptosis under ER stress conditions.

\section{ROS Up-Regulates ER Stress in HT29-H and HT29-L Cells}

Inhibiting ROS with DPI before, during, or after tunicamycin treatment not only inhibited apoptosis but also

Table 1 Morphology of Goblet Cells from Mouse Colons during the Progressive Development of DSS-Induced Colitis

\begin{tabular}{llll}
\hline & \multicolumn{3}{l}{ Total goblet cells, \% } \\
\cline { 2 - 4 } DSS treatment & Filled & Releasing mucus & Empty \\
\hline Water controls & $60.2 \pm 7.7$ & $38.6 \pm 7.7$ & $1.2 \pm 0.1$ \\
Day 1 & $41.9 \pm 4.8$ & $57.6 \pm 4.6$ & $0.6 \pm 0.3$ \\
Day 2 & $28.8 \pm 4.9^{*}$ & $57.4 \pm 2.3$ & $13.8 \pm 2.6^{* *}$ \\
Day 3 & $19 \pm 3.1^{* *}$ & $52.8 \pm 3.4$ & $28.2 \pm 1.7^{* * *}$ \\
Day 4 & $14.8 \pm 3.5^{* *}$ & $85.2 \pm 3.5^{* *}$ & ND \\
Day 6 & $6.4 \pm 0.6^{* *}$ & $93.6 \pm 0.6^{* *}$ & ND \\
\hline
\end{tabular}

Tissue sections were stained with periodic acid-Schiff to quantify goblet cell morphology. A minimum of 100 goblet cells were counted from each section. Goblet cells were graded as filled with mucin, actively releasing mucin, or empty goblet cells (few mucin granules) at each day during DSS-induced colitis. Data are expressed as \% of goblet cells \pm SEM from 5 animals per day.

${ }^{*} P<0.05,{ }^{*} P<0.01$, and ${ }^{* * *} P<0.001$ versus the water control group.

DSS, dextran sulfate sodium; ND, not determined (because the epithelium layer was severely damaged). markedly inhibited ER stress (Figure 6A). Pretreatment and simultaneous treatments were most effective, whereas posttreatment only slightly reduced ER stress. Similarly, DPI alleviated thapsigargin-induced ER stress (Figure 6B), suggesting that ROS is a major factor that up-regulated ER stress. Consistent with this, HT29-H produced significantly more ROS basally than HT29-L cells in a temporal manner (Figure 6C), suggesting that high MUC2 biosynthesis directly increases ROS production, which, in turn, elevated ER stress. It was next explored whether high ROS production in HT29$\mathrm{H}$ versus HT29-L cells was because of higher mitochondrial numbers in the former versus the latter. Both cell types had similar mitochondria mass (Figure 6D), but ATP production, a measure of mitochondrial activity, was significantly higher in HT29-H than HT29-L cells (Figure 6E), suggesting that the high MUC2 demand in HT29-H cells caused the mitochondria to be more metabolically active than HT29-L cells. These data show that high MUC2 production increases ROS production that drives ER stress. Because ER stress can induce ROS production, ${ }^{32}$ ER stress was induced with tunicamycin and thapsigargin. Tunicamycin treatment significantly increased ROS in both cell lines, whereas treatment with thapsigargin only slightly increased ROS in HT29-H cells (Figure 6, F and G). As expected, inhibiting ER stress with TUDCA significantly reduced ROS production basally and after treatment with tunicamycin (Figure $6 \mathrm{H}$ ), suggesting a cycle in goblet cells where MUC2-induced ROS increases ER stress and vice versa.

\section{ER Stress Decreases MUC2 Production and Disrupts Barrier Function in Vitro}

In goblet cells, the accumulation of misfolded MUC2 because of ER stress reduced the production and secretion of mature MUC2 (Figure 2A) that can subsequently disrupt barrier function. This was interrogated by inducing ER stress with tunicamycin, and its effect was analyzed on barrier dysfunction. ER stress significantly decreased MUC2 production in both HT29-H/L cells (Figure 6I). To explore if loss of secreted MUC2 affected epithelial barrier function, cells were exposed to the colonic protozoan parasite E. histolytica. Entamoeba histolytica lives in the colonic mucus layer that forms the first line of innate host defense by preventing the parasite from invading the underlying colonic epithelium. ${ }^{33,34}$ In nonstressed cells, $E$. histolytica destroyed $54 \%$ of HT29-H monolayer and $70 \%$ of HT29-L cells, consistent with more mucus production and protection in HT29-H than HT29-L cells. However, after treatment with tunicamycin that decreased mature MUC2 production, E. histolytica readily attached to and destroyed $79 \%$ of HT29-H (25\% more than untreated cells) and $82 \%$ of HT29-L (12\% more than untreated cells) cells. More important, HT29-H cells became as susceptible to $E$. histolytica-induced monolayer destruction as HT29-L cells under ER stress conditions (Figure $6 \mathrm{~J}$ ). These results clearly demonstrate that MUC2 is protective on the epithelia and 

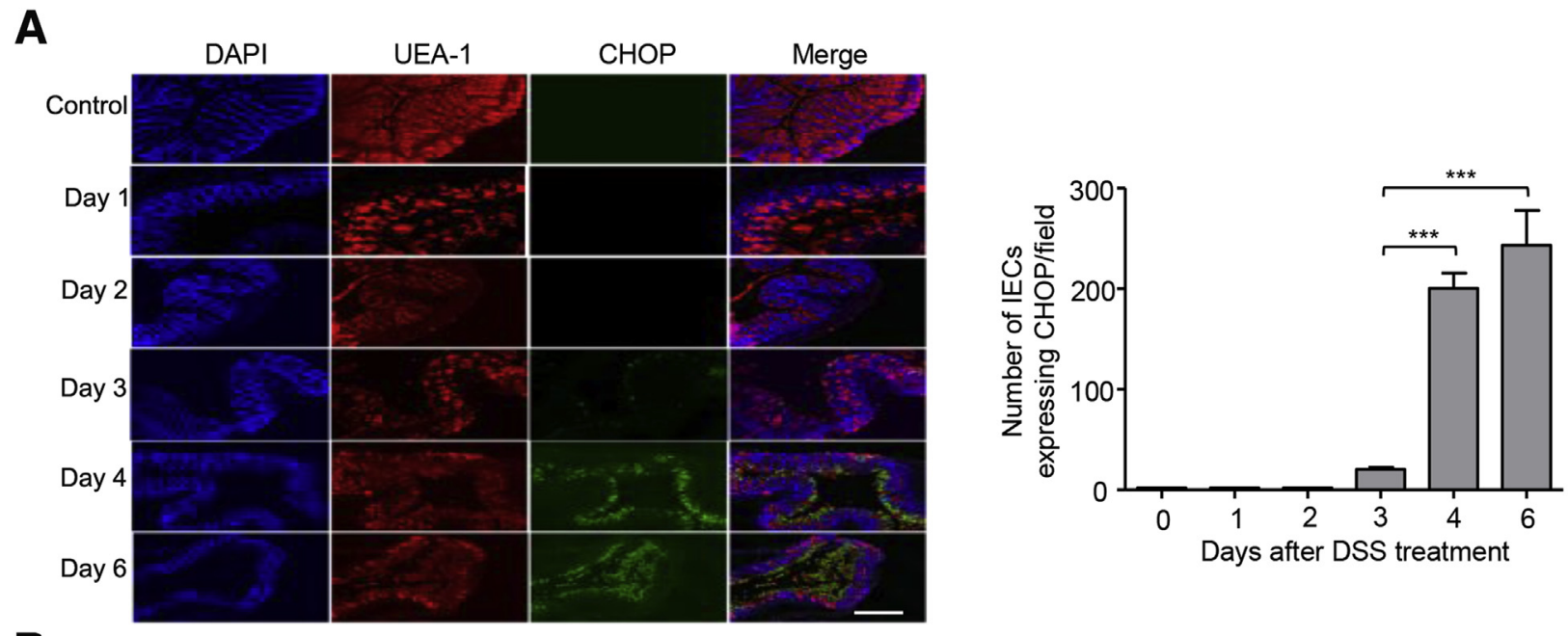

B
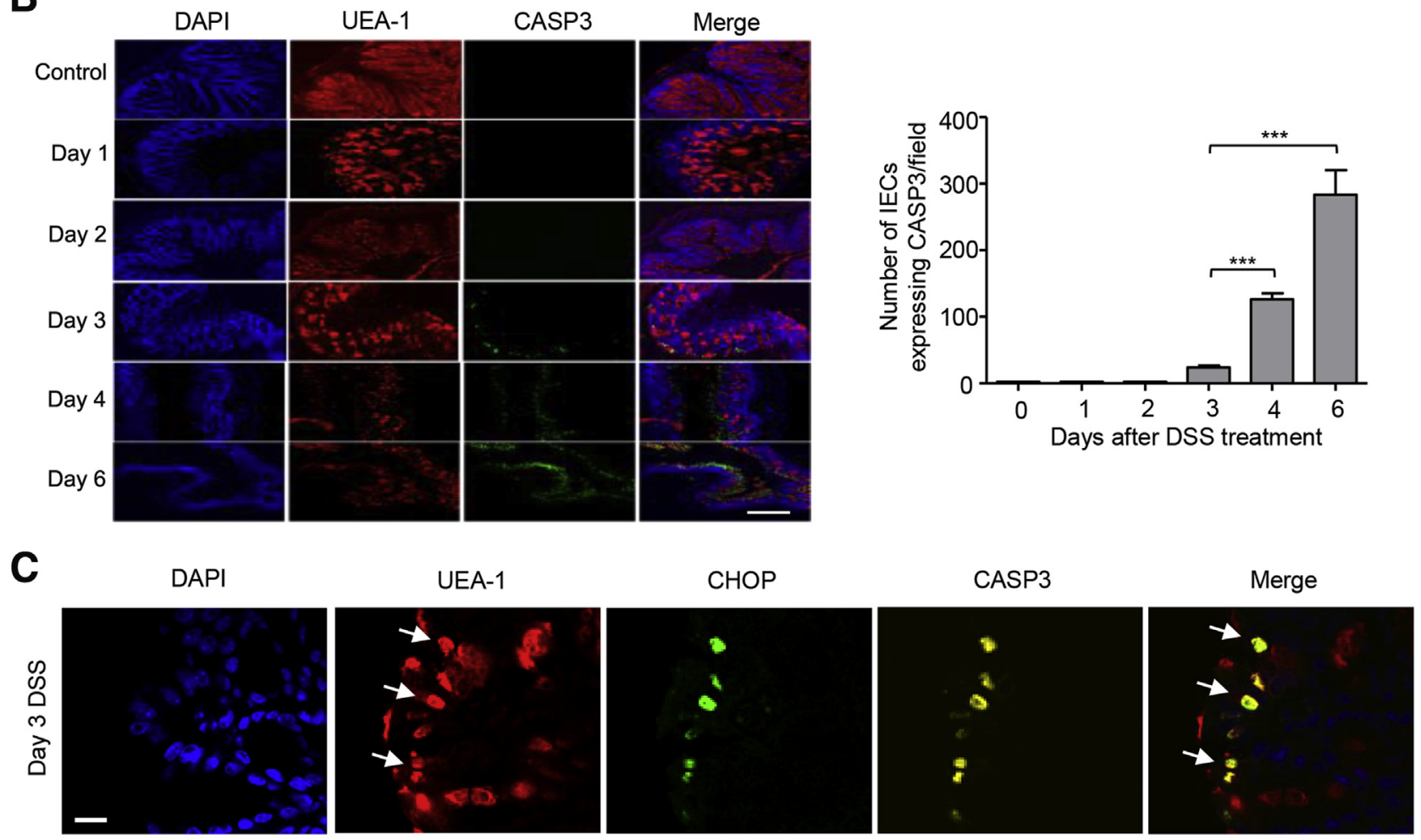

D
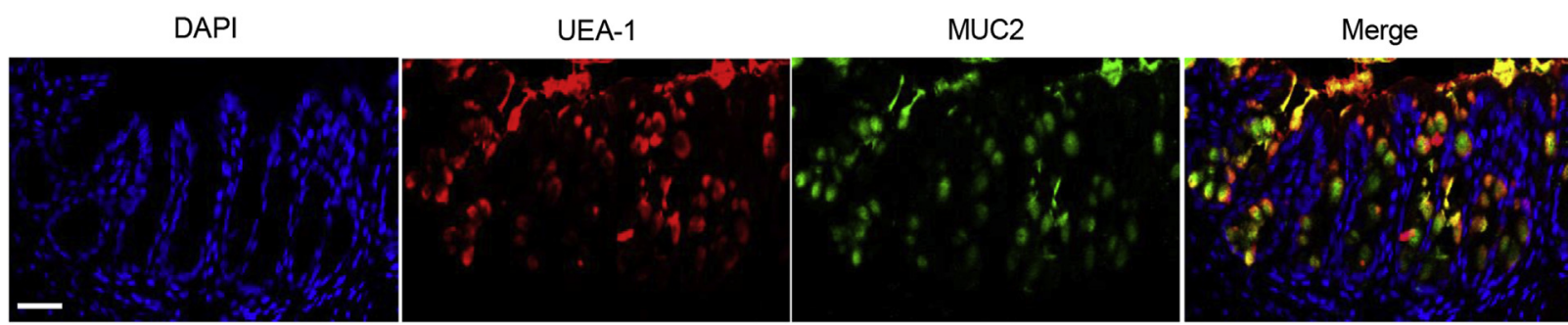

Figure 8 Goblet cells preferentially undergo endoplasmic reticulum (ER) stress and apoptosis during dextran sulfate sodium (DSS)-induced colitis. A and B: Colon tissues were stained with antibodies that recognize mucins in goblet cells [Ulex europaeus agglutinin 1 (UEA-1)], CHOP, and cleaved caspase 3 (CASP3). Expression of proteins was determined by confocal microscopy. The number of colonic epithelial cells undergoing apoptosis was quantified by comparing the number of cleaved caspase $3-$ and CHOP-positive cells with total numbers of epithelial cells. C: Colon tissues from mice at day 3 of DSS administration were stained with DAPI to detect nuclei, UEA-1 to visualize mucins in goblet cells, and CHOP and cleaved caspase 3 to detect ER stress-induced apoptosis. Arrows show goblet cells undergoing ER stress and apoptosis. D: Colon tissues from mice were stained with DAPI to detect nuclei, UEA-1, and antiMUC2 antibody to visualize MUC2 in goblet cells. Data are expressed as means \pm SEM (A and $\mathbf{B}) . n=4$ mice (A and $\mathbf{B}$ ). ${ }^{* * * P}<0.001$ (analysis of variance with Bonferroni's post hoc test). Scale bars $=10 \mu \mathrm{m}(\mathbf{A}-\mathbf{D})$. CHOP, CCAAT-enhancer-binding protein homologous protein; IEC, intestinal epithelial cells. 
A

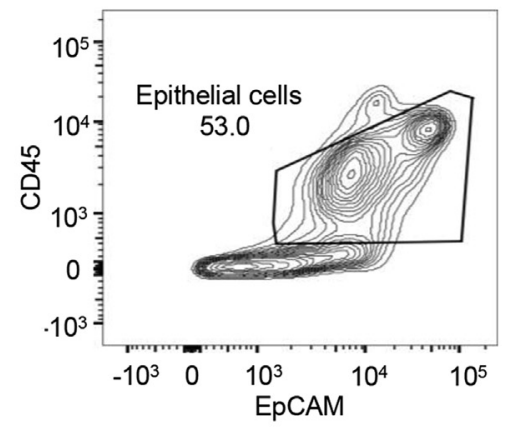

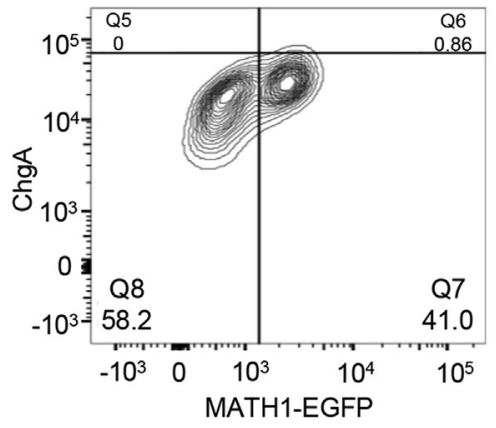

B

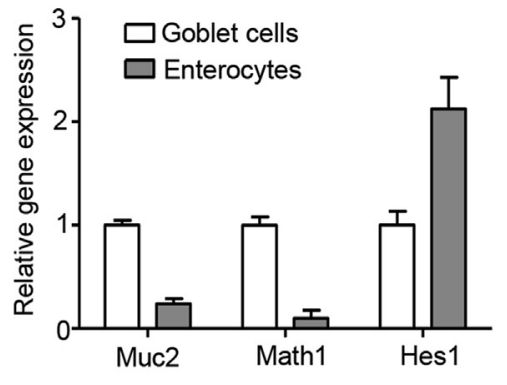

C

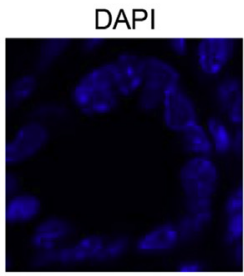

UEA-1
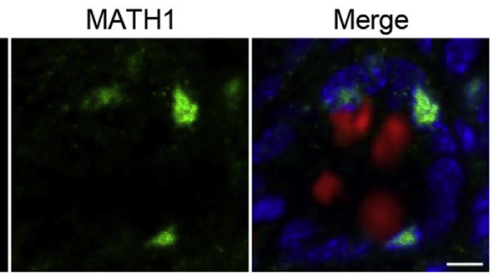

D
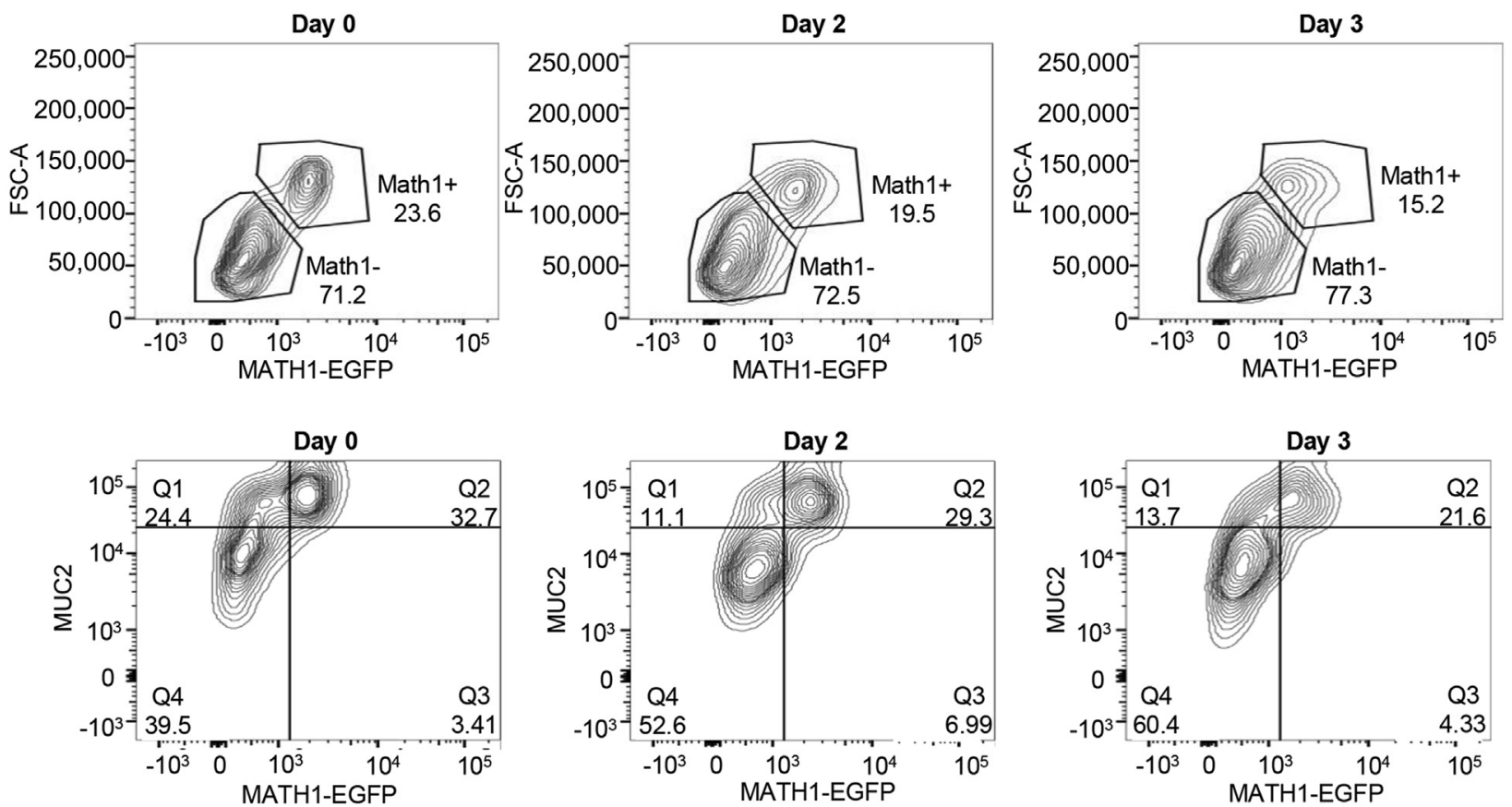

Day 3
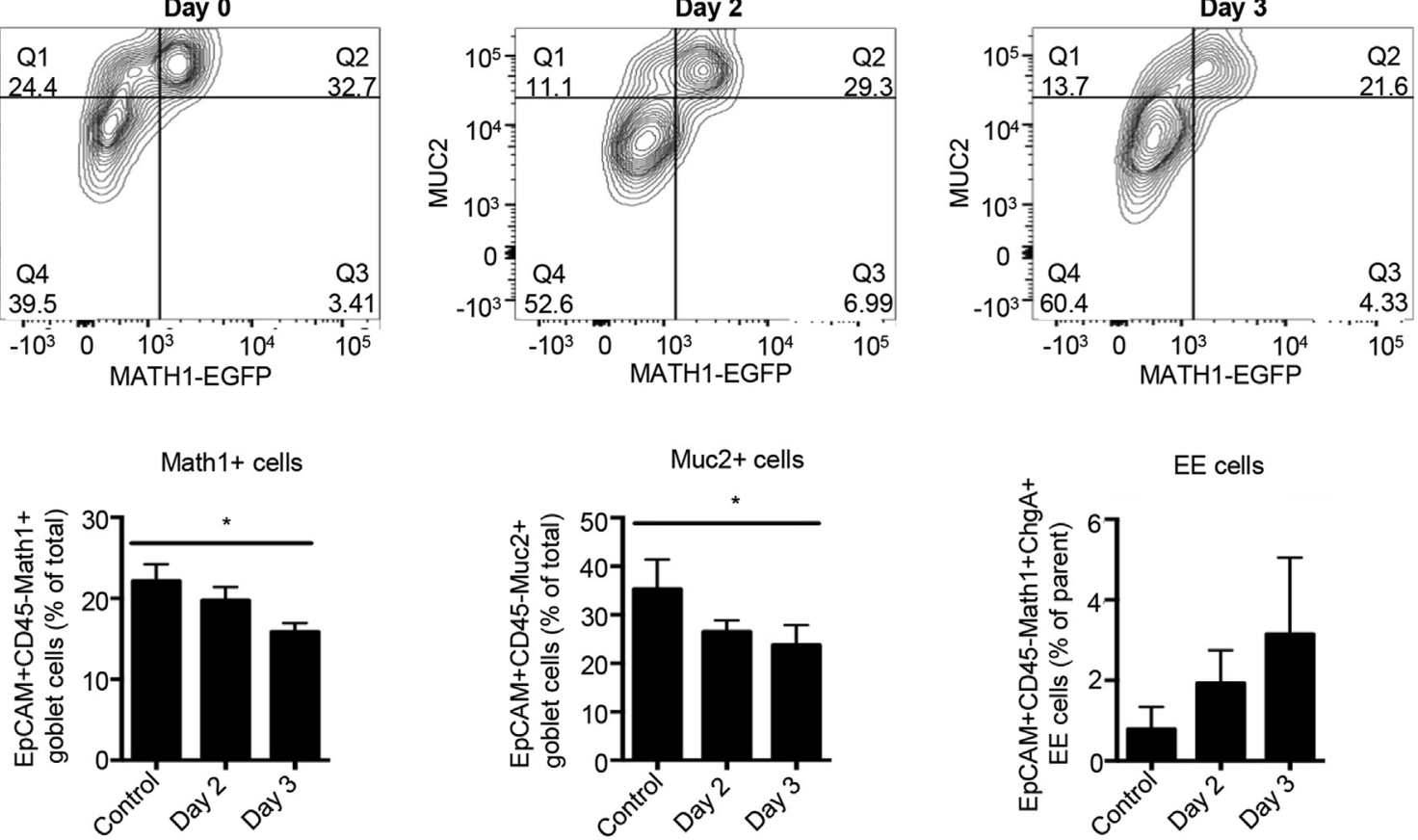
ER stress can lead to loss of mature MUC2 mucin, thus limiting its barrier property and increasing its susceptibility to epithelial invasion by pathogens.

\section{Goblet Cells Preferentially Undergo Apoptosis in DSS-Induced Colitis}

Consistent with the previous study, it was determined if ER stress can lead to loss of Muc2 production and barrier dysfunction in DSS-induced colitis. ${ }^{35} \mathrm{Muc}^{+/+}$mice were administered $3.5 \%$ DSS in drinking water for 5 days, followed by water. Weight loss was observed by day 6 (approximately 6\%), which continued up to day 9, with approximately $19 \%$ body weight loss. Because DSS administered on the mucosal side may cause ER stress at the onset of colitis, ER stress responses during the first 6 days were studied. Isolated colonic tissues showed a temporal increase in proinflammatory cytokines tumor necrosis factor- $\alpha$, interferon- $\gamma$, and IL-1 $\beta$ that coincided with progression of DSS-induced colitis. In parallel, there was a significant timedependent up-regulation of ER stress genes, GRP78, ATF4, and $C H O P$, which was consistent with the induction of proinflammatory cytokines as early as days 2 to 3 (Figure 7, A-C). In $M u c 2^{-/-}$mice, proinflammatory cytokines and ER stress markers were only modestly increased in response to $1 \%$ DSS (Supplemental Figure S3). In particular, on days 4 and 6 , when tissue ulcerations were most severe, proinflammatory cytokine levels and ER stress markers were significantly lower than those elicited in $\mathrm{Muc}^{+/+}$littermates. $M u c 2^{-/}$mice are highly susceptible to DSS-induced colitis and are prone to development of colitis and cancers. ${ }^{36,37}$

Because high MUC2 production by goblet cells renders them more sensitive to ER stress and apoptosis than absorptive epithelial cells (Figure 1J), it was next investigated whether goblet cells undergo apoptosis during earlyonset colitis. The number of goblet cells in the colon that were filled with mucins, those actively releasing mucins, and those that were empty in DSS-treated mice were quantified. Controls as well as day 1 and 2 DSS-treated mice had well-organized crypts with mucin-filled goblet cells (Figure 7D). In control animals, $60 \%$ of goblet cells were filled with mucin, $39 \%$ were releasing mucins, and only $1 \%$ were empty (Table 1). On day 1 of DSS treatment, there was considerable mucin secretagogue activity, with $58 \%$ of goblet cells actively releasing mucins, $42 \%$ filled with mucins, and only $0.6 \%$ empty. Starting on day 2 , mucin secretagogue activity resulted in significantly reduced mucin-filled goblet cells; by days 4 and 6 of DSS treatment, mucosal lesions were observed and goblet cells were undetectable in the damaged area. Areas adjacent to the lesions had few goblet cells present, with a paucity of periodic acidSchiff-positive mucin (Figure 7D and Table 1).

To determine whether loss of mucin-filled periodic acidSchiff-positive cells was because of ER stress-induced apoptosis, the goblet cells were quantified by confocal immunofluorescence microscopy. CHOP and cleaved caspase 3 proteins were not detected in control mice on days 1 and 2 of DSS treatment. However, as early as day 3, a few epithelial cells showed expression of both CHOP and cleaved caspase 3 . Thereafter, epithelial cells expressing these proteins significantly increased on days 4 and 6 (Figure 8, A and B). The increase in ER stress and apoptosis coincided with significant decrease in mucin-filled goblet cells. Because day 3 was the earliest time point when ER stress and apoptosis were observed, it was of interest to determine whether the cells undergoing apoptosis were goblet cells or other enterocytes. Confocal microscopy revealed colocalization of CHOP, cleaved caspase 3, and tetrarhodamine isothiocyanate-conjugated Ulex europaeus agglutinin 1 (stains fucose in mucins) in goblet cells, whereas other enterocytes were not undergoing ER stress or apoptosis (Figure 8, C and D). To validate and quantify the microscopy findings, colonic epithelial cells from Math1 ${ }^{\text {M1GFP }}$ mice were analyzed and fluorescence-activated cell sorted to specifically differentiate between enterocyte and goblet cell populations. In the colonic epithelium, Math1 is expressed exclusively by secretory cells, and its ablation leads to a complete loss of goblet cell lineage. ${ }^{38}$ Isolated mouse epithelial cells were fluorescence-activated cell sorted on the basis of a lack of CD45, a common immune cell marker, and presence of epithelial cell adhesion molecule (EpCAM), which is only expressed by epithelial cells. Colonic $\mathrm{CD} 45^{-} \mathrm{EpCAM}^{+}$cells were partitioned into cells that were Math1 ${ }^{\text {M1GFP }}$ negative (enterocytes) and Math $1^{\text {M1GFP }}$ positive [enteroendocrine (EE) cells and goblet cells]. EE cells were separated from goblet cells by staining with MUC2 (positive for goblet cells and negative for EE cells) and chromogranin A (positive for EE cells and negative for goblet cells). The percentage of EE cells was relatively small $(0.86 \%)$ in the colon compared with the

Figure 9 Validation of goblet cell loss during dextran sulfate sodium (DSS)-induced colitis. A: Flow cytometry analysis showing the gating strategy used to distinguish enterocyte $\left(\mathrm{CD}_{5} 5^{-} \mathrm{EpCAM}^{+}\right)$and nonenterocyte $\left(\mathrm{CD}^{+} 5^{+} \mathrm{EpCAM}^{-}\right)$populations from isolated mouse (Math $1^{\mathrm{M} 1 \mathrm{GFP}}$ ) colon epithelial cells. $\mathrm{CD}^{-} 5^{-} \mathrm{EpCAM}^{+}$cells were then separated into cells that were Math $1^{\text {M1GFP }}$ positive, chromogranin A (ChgA) negative (goblet cells), and Math ${ }^{\text {M1GFP }}$ negative (enterocytes). B: The cells were sorted into goblet cell and enterocyte populations and validated by real-time quantitative PCR for MUC2, MATH1, and HES1. Gene expressions were normalized to $\beta$-actin as housekeeping gene. C: Colon tissues from Math ${ }^{\text {M1GFP }}$ mice were stained with antibodies to show expression of MATH1 by goblet cells [Ulex europaeus agglutinin $1(\mathrm{UEA}-1)^{+}$cells]. D: Math ${ }^{\mathrm{M} 1 \mathrm{GFP}}$ mice were administered $3.5 \%$ DSS, and colonic epithelial cells were isolated on days 2 and 3. The isolated cells were stained with CD45, EpCAM, ChgA, and MUC2 antibodies and subjected to flow cytometric analysis. Colonic

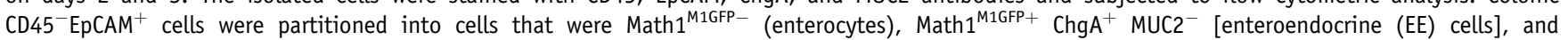
Math $1^{\text {M1GFP }+} \mathrm{ChgA}^{-}$MUC2 $^{+}$(goblet cells), and percentages of enterocyte, EE cell, and goblet cell populations were quantified. ${ }^{*} P<0.05$. Scale bar $=10 \mu \mathrm{m}$ (C). EGFP, enhanced green fluorescent protein; EpCAM, epithelial cell adhesion molecule; FSC, forward scatter. 


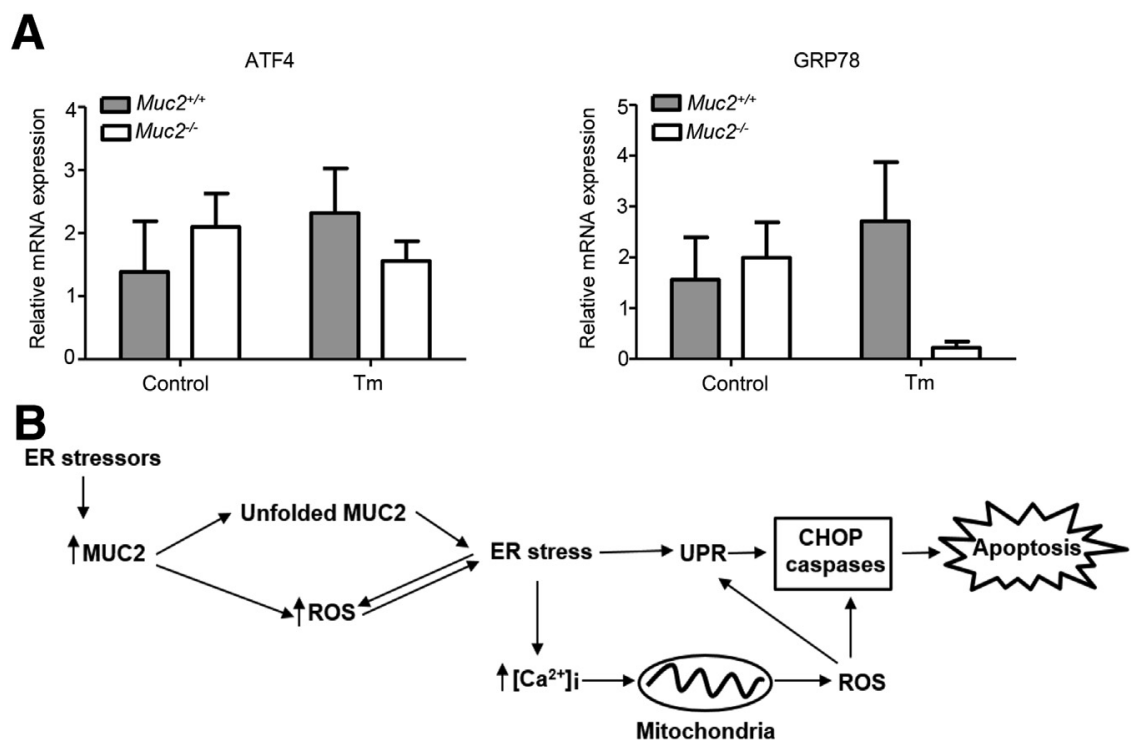

Figure 10 A: Colonic organoids from $\mathrm{MuC2}^{+/+}$ are more susceptible to endoplasmic reticulum (ER) stress than $\mathrm{MuC2}^{-1-}$ littermates. Organoids prepared from the colons of $\mathrm{MuC2}^{+/+}$and $\mathrm{MuC2}^{-/-}$littermates were treated with $10 \mu \mathrm{g} / \mathrm{mL}$ tunicamycin $(\mathrm{Tm})$ for 6 hours, and ATF4 and GRP78 gene expression levels were determined by real-time quantitative PCR. Gene expression levels were normalized using GAPDH as housekeeping gene. B: Proposed role of high MUC2 biosynthesis in goblet cell apoptosis. High MUC2 production increases misfolded MUC2 and reactive oxygen species (ROS) production, which leads to ER stress. ER stress, in turn, activates the unfolded protein response (UPR) and increases intracellular calcium, which induces the mitochondria to elevate ROS production. Increase in ROS production then activates proapoptotic proteins ( $\mathrm{CHOP}$ and caspases) to cause apoptosis. Data are expressed as means $\pm \operatorname{SEM}(\mathbf{A}) . n=2$ separate experiments $(\mathbf{A})$. ATF, activating transcription factor mRNA expression; $\left[\mathrm{Ca}^{2+}\right] \mathrm{i}$, intracellular calcium levels; CHOP, CCAATenhancer-binding protein homologous protein; GRP, glucose-regulated protein mRNA expression.

percentage of goblet cells (41\%). To confirm the identity of these populations, sorted cells were analyzed by real-time quantitative PCR for common markers. Enterocytes expressed elevated levels of Hes1, the transcription factor critical for absorptive lineage. Conversely, goblet cells expressed greater levels of Math1 and Muc2 compared with enterocytes (Figure 9, A-C). To study the depletion of goblet cells, animals were given DSS for 2 to 3 days and colonic epithelial cells were sorted. In agreement with the histologic findings, there was rapid decrease in Math1- and MUC2-positive goblet cells after DSS; however, abundance of enterocytes and EE cells was not affected (Figure 9D). DSS was not selectively toxic to goblet cells because in vitro treatment of HT29-H/L and Caco-2 cells with DSS showed equal susceptibility to ER stress and apoptosis. These data clearly demonstrate that Muc2-positive goblet cells were significantly reduced in the early stages of colitis because of preferential death via ER stress and apoptosis. To determine whether goblet cells were more sensitive to ER stress, organoids prepared from the colons of $\mathrm{Muc2}^{+/+}$and $M u c 2^{-1-}$ littermates (lacking periodic acid-Schiff-positive goblet cells) were treated with tunicamycin. Under ER stress conditions, ER stress markers in organoids were elevated in $\mathrm{Muc2}^{+/+}$mice and remained unaltered (ATF4) or reduced (GRP78) in $\mathrm{Muc}^{-\prime-}$ mice (Figure 10A). A hypothetical model is proposed to depict how high MUC2 biosynthesis can lead to goblet cell apoptosis (Figure 10B).

\section{Discussion}

It is well known that alterations in MUC2 biosynthesis and structure/function severely affect epithelial barrier function that can lead to bacterial invasion and/or translocation of noxious substances into the systemic circulation during inflammation. ${ }^{12}$ In ulcerative colitis, goblet cell depletion is commonly characterized by a thin/depleted mucus layer and a dysfunctional mucosal barrier. Surprisingly, the mechanism of goblet cell depletion and the role of MUC2 biosynthesis in this phenomenon have not been explored. It is unclear if goblet cell depletion refers to loss of MUC2 mucin and/or loss of goblet cells. Herein, we conclusively show that high MUC2 production increases goblet cell sensitivity to ER stress and apoptosis, thus causing preferential loss of goblet cells in the colonic epithelium during DSS-induced colitis. These findings were corroborated in vitro and showed high MUC2 output specifically increases ROS production that drives ER stress and apoptosis in goblet cells.

The high MUC2 phenotype exhibited significantly higher susceptibility to ER stress and apoptosis compared with the low MUC2 phenotype, showing that high MUC2 biosynthesis increases goblet cell sensitivity to death in the presence of ER stress/apoptosis stimuli. MUC2 is a large glycoprotein, and the complexity of its synthesis makes it prone to misfolding in the ER, leading to ER stress. In IBD, goblet cells are constantly exposed to ER stress or other harmful stimuli present in the lumen and are prone to death attributable to ER stress. As predicted, DSS-induced colitis in $\mathrm{Muc}^{+/+}$littermates elicited a robust time-dependent increase in proinflammatory cytokine and ER stress responses and goblet cells exhibited severe ER stress and subsequent apoptosis during the early stages of colitis. More important, goblet cells were the first cells to undergo apoptosis compared with other enterocytes, as revealed by confocal microscopy, which was consistent with the in vitro data that showed that non-MUC2-producing Caco-2 cells are highly resistant 
to ER stress. In comparison, DSS-induced colitis in $M u c 2^{-1-}$ littermates showed significantly lower proinflammatory cytokine and ER stress responses. To corroborate these findings, Math $1^{\mathrm{M} 1 \mathrm{GFP}}$ mice that express green fluorescent protein (GFP) in goblet cells were used. Because there is no extracellular marker to differentiate between goblet cells and enterocytes, the GFP marker was critical to fluorescence-activated cell-sorted goblet cells. As predicted, flow cytometric analysis of colonic epithelial cells isolated from Math $1^{\text {M1GFP }}$ mice showed that the goblet cell loss (decrease in mean fluorescence intensity and loss of $\mathrm{GFP}^{+}$goblet cell population) occurred as early as day 2 of DSS administration, whereas the enterocyte population remained relatively unchanged. These findings demonstrate that goblet cell apoptosis is one of the initial inciting events that leads to the loss of the protective mucus barrier in the pathogenesis of colitis. Moreover, goblet cell apoptosis can potentially generate portals for entry of enteric pathogens during early-onset colitis.

MUC2-induced ER stress has recently been linked to the pathogenesis of IBD. For example, missense mutation in Muc2 in the N-ethyl N-nitrosourea mutants Winnie and Eeyore mice was shown to cause aberrant Muc2 misfolding, leading to ER stress and spontaneous inflammation, similar to human ulcerative colitis. ${ }^{14,39,40}$ In addition, mice deficient in mucin-folding enzymes, such as mucin-specific protein disulfide isomerases ${ }^{15}$ and fatty acid synthases, ${ }^{16}$ develop spontaneous colitis because of ER stress caused by misfolded Muc2. Colonic tissues from patients with IBD have also showed increased ER stress markers. ${ }^{41,42}$ These studies of ER stress were conducted in the context of MUC2 mutations and loss of MUC2-specific folding enzymes in goblet cells. Our studies provide compelling evidence that not only do enzyme deficiencies or mutations in MUC2 cause ER stress, but also increased MUC2 output by goblet cells under inflammatory and disease states can lead to this condition. Increased MUC2 output can be triggered when goblet cells are exposed to microbial factors and proinflammatory cytokines in IBD, which can lead to increased MUC2 misfolding and eventual ER stress and apoptosis of goblet cells. So, although high MUC2 production is not a primary factor in IBD pathogenesis, it is a major factor that leads to progression of the disease. The chemical chaperone TUDCA reversed ER stress and rescued goblet cells from apoptosis, which suggests that enhancing MUC2 folding by developing chemical chaperones for therapeutic use can preserve goblet cells and help to maintain the integrity of the mucus barrier. A recent study $^{43}$ has shown that TUDCA and 4-phenylbutyrate facilitate protein folding and reduce ER stress and colitis in mice.

ROS are highly reactive molecules that cause tissue damage when their cytotoxic effects overwhelm scavenging by antioxidants. ROS are known to cause ER stress in several cell types, ${ }^{44,45}$ and oxidative stress has been linked to various diseases, including IBD. ${ }^{46,47}$ In this study, ROS played a critical role in goblet cell ER stress and apoptosis. Inhibition of ROS markedly inhibited ER stress and alleviated apoptosis by inhibiting various apoptotic events. Furthermore, the high mucin phenotype produced significantly more ROS than the low mucin phenotype. This shows that high MUC2 output is associated with increased ROS production that subsequently contributes to misfolding in the ER. Inhibiting tunicamycin-induced ER stress with TUDCA significantly reduced ROS production, suggesting that misfolded proteins also cause increased ROS production. Therefore, there appears to be a positive feedback loop within goblet cells where ROS can induce ER stress/protein misfolding and vice versa. However, the fact that TUDCA significantly inhibited basal ROS levels may suggest that the chaperone enhances MUC2 folding by inhibiting ROS production; thus, ROS may be the initiating factor in the cycle. Increased ROS production in the high mucin phenotype was because of enhanced mitochondrial activity, although mitochondrial mass was similar in both cell types. Mitochondria are a major source of ROS and play an important role in ER stress-induced apoptosis. ${ }^{48}$ It is known that accumulation of unfolded proteins in the ER causes calcium leakage into the cytosol, increasing ROS production in the mitochondria. ${ }^{48}$ In addition, because protein folding in the ER lumen is a highly energydependent process, ATP depletion attributable to protein misfolding triggers mitochondrial oxidative phosphorylation to increase ATP and ROS production above threshold levels, ${ }^{49}$ leading to apoptosis. This is consistent with our findings in which high MUC2 production resulted in higher ATP and ROS production.

In summary, this study shows that high MUC2 output increases goblet cell ER stress and apoptosis in the presence of external stimuli analogous to IBD, where mucosal epithelial cells are constantly exposed to microbial and noxious stimuli that drive ER stress. These studies also reveal that goblet cells are highly sensitive to stressors preferentially causing them to undergo apoptosis during early-onset colitis, and this may be one of the early events that perpetuates progression of the disease. Developing drugs and/or biological agents that enhance MUC2 folding can thus help to alleviate goblet cell depletion, maintain mucosal barrier integrity, and better manage IBD.

\section{Acknowledgments}

We thank Pina Colarusso and Jen Amon from the live cell imaging facility (Snyder Institute) for technical assistance, Prof. Michael McGuckin (University of Queensland, South Brisbane, QLD, Australia) for the 4F1 monoclonal antibody, and Dr. Christian Laboisse (Université de Nantes, Nantes, France) for HT29-H cells. 
A.T., S.C., and K.C. conceived and designed the study, acquired, analyzed, and interpreted data, and drafted the manuscript; A.T., F.M., S.C., H.G., M.K., and S.T. performed the experiments and statistical analysis; A.T., F.M., S.C., and K.C. contributed reagents, materials, and analysis tools; K.C. obtained funding and supervised the study.

\section{Supplemental Data}

Supplemental material for this article can be found at https://doi.org/10.1016/j.ajpath.2018.02.007.

\section{References}

1. McCracken VJ, Lorenz RG: The gastrointestinal ecosystem: a precarious alliance among epithelium, immunity and microbiota. Cell Microbiol 2001, 3:1-11

2. Gum JR, Hicks JW, Gillespie AM, Carlson EJ, Kömüves L, Karnik S, Hong JC, Epstein CJ, Kim YS: Goblet cell-specific expression mediated by the MUC2 mucin gene promoter in the intestine of transgenic mice. Am J Physiol 1999, 276:G666-G676

3. Johansson ME, Phillipson M, Petersson J, Velcich A, Holm L, Hansson GC: The inner of the two Muc2 mucin-dependent mucus layers in colon is devoid of bacteria. Proc Natl Acad Sci U S A 2008, 105:15064-15069

4. Cone RA: Barrier properties of mucus. Adv Drug Deliv Rev 2009, 61: $75-85$

5. Hollingsworth MA, Swanson BJ: Mucins in cancer: protection and control of the cell surface. Nat Rev Cancer 2004, 4:45-60

6. Boltin D, Perets TT, Vilkin A, Niv Y: Mucin function in inflammatory bowel disease, an update. J Clin Gastroenterol 2013, 47:106-111

7. Corfield AP, Carroll D, Myerscough N, Probert CS: Mucins in the gastrointestinal tract in health and disease. Front Biosci 2001, 6: D1321-D1357

8. Backstrom M, Ambort D, Thomsson E, Johansson ME, Hansson GC: Increased understanding of the biochemistry and biosynthesis of MUC2 and other gel-forming mucins through the recombinant expression of their protein domains. Mol Biotechnol 2013, 54:250-256

9. Johansson MEV, Hansson GC: Mucus and the goblet cell. Dig Dis 2013, 31:305-309

10. Godl K, Johansson ME, Lidell ME, Mörgelin M, Karlsson H, Olson FJ, Gum JR Jr, Kim YS, Hansson GC: The N terminus of the MUC2 mucin forms trimers that are held together within a trypsinresistant core fragment. J Biol Chem 2002, 277:47248-47256

11. Ambort D, Johansson MEV, Gustafsson JK, Nilsson HE, Ermund A, Johansson BR, Koeck PJ, Hebert H, Hansson GC: Calcium and pHdependent packing and release of the gel-forming MUC2 mucin. Proc Natl Acad Sci U S A 2012, 109:5645-5650

12. Vereecke L, Beyaert R, Van Loo G: Enterocyte death and intestinal barrier maintenance in homeostasis and disease. Trends Mol Med 2011, 17:584-593

13. Kim YS, Ho SB: Intestinal goblet cells and mucins in health and disease: recent insights and progress. Curr Gastroenterol Rep 2010, 12 : 319-330

14. Heazlewood CK, Cook MC, Eri R, Price GR, Tauro SB, Taupin D, Thornton DJ, Png CW, Crockford TL, Cornall RJ, Adams R, Kato M, Nelms KA, Hong NA, Florin TH, Goodnow CC, McGuckin MA: Aberrant mucin assembly in mice causes endoplasmic reticulum stress and spontaneous inflammation resembling ulcerative colitis. PLoS Med 2008, 5:440-460

15. Park SW, Zhen G, Verhaeghe C, Nakagami Y, Nguyenvu LT, Barczak AJ, Killeen N, Erle DJ: The protein disulfide isomerase AGR2 is essential for production of intestinal mucus. Proc Natl Acad Sci U S A 2009, 106:6950-6955

16. Wei X, Yang Z, Rey FE, Ridaura VK, Davidson NO, Gordon JI, Semenkovich CF: Fatty acid synthase modulates intestinal barrier function through palmitoylation of mucin 2. Cell Host Microbe 2012, $11: 140-152$

17. Jäger R, Bertrand MJ, Gorman AM, Vandenabeele P, Samali A: The unfolded protein response at the crossroads of cellular life and death during endoplasmic reticulum stress. Biol Cell 2012, 104:259-270

18. Szegezdi E, Logue SE, Gorman AM, Samali A: Mediators of endoplasmic reticulum stress-induced apoptosis. EMBO Rep 2006, 7:880-885

19. Akiba Y, Guth PH, Engel E, Nastaskin I, Kaunitz JD: Dynamic regulation of mucus gel thickness in rat duodenum. Am J Physiol Gastrointest Liver Physiol 2000, 279:G437-G447

20. Augeron C, Laboisse CL: Emergence of permanently differentiated cell clones in a human colonic cancer cell line in culture after treatment with sodium butyrate. Cancer Res 1984, 44:3961-3969

21. Belley A, Keller K, Grove J, Chadee K: Interaction of LS174T human colon cancer cell mucins with Entamoeba histolytica: an in vitro model for colonic disease. Gastroenterology 1996, 111:1484-1492

22. Devine PL, McGuckin MA, Birrell GW, Whitehead RH, Sachdev GP, Shield P, Ward BG: Monoclonal antibodies reacting with the MUC2 mucin core protein. Br J Cancer 1993, 67:1182-1188

23. Cobo ER, Kissoon-Singh V, Moreau F, Chadee K: Colonic MUC2 mucin regulates the expression and antimicrobial activity of $\beta$-defensin 2. Mucosal Immunol 2015, 8:1360-1372

24. O'Connell J, Bennett MW, Nally K, O'Sullivan GC, Collins JK, Shanahan F: Interferon- $\gamma$ sensitizes colonic epithelial cell lines to physiological and therapeutic inducers of colonocyte apoptosis. J Cell Physiol 2000, 185:331-338

25. McGuckin MA, Eri RD, Das I, Lourie R, Florin TH: ER stress and the unfolded protein response in intestinal inflammation. Am J Physiol Gastrointest Liver Physiol 2010, 298:G820-G832

26. Gaudier E, Jarry A, Blottière HM, de Coppet P, Buisine MP, Aubert JP, Laboisse C, Cherbut C, Hoebler C: Butyrate specifically modulates MUC gene expression in intestinal epithelial goblet cells deprived of glucose. Am J Physiol Gastrointest Liver Physiol 2004, 287:G1168-G1174

27. Higa A, Mulot A, Delom F, Bouchecareilh M, Nguyên DT, Boismenu D, Wise MJ, Chevet E: Role of pro-oncogenic protein disulfide isomerase (PDI) family member anterior gradient 2 (AGR2) in the control of endoplasmic reticulum homeostasis. J Biol Chem 2011, 286:44855-44868

28. Higa A, Chevet E: Redox signaling loops in the unfolded protein response. Cell Signal 2012, 24:1548-1555

29. Kurihara Y, Kanki T, Aoki Y, Hirota Y, Saigusa T, Uchiumi T, Kang D: Mitophagy plays an essential role in reducing mitochondrial production of reactive oxygen species and mutation of mitochondrial DNA by maintaining mitochondrial quantity and quality in yeast. $\mathrm{J}$ Biol Chem 2012, 287:3265-3272

30. Hitomi J, Katayama T, Eguchi Y, Kudo T, Taniguchi M, Koyama Y, Manabe $T$, Yamagishi $S$, Bando $Y$, Imaizumi $K$, Tsujimoto $Y$ Tohyama M: Involvement of caspase-4 in endoplasmic reticulum stress-induced apoptosis and Abeta-induced cell death. J Cell Biol 2004, 165:347-356

31. Nakagawa T, Zhu H, Morishima N, Li E, Xu J, Yankner BA, Yuan J: Caspase-12 mediates endoplasmic-reticulum-specific apoptosis and cytotoxicity by amyloid $\beta$. Nature 2000, 403:98-103

32. Haynes CM, Titus EA, Cooper AA: Degradation of misfolded proteins prevents ER-derived oxidative stress and cell death. Mol Cell 2004, 15 767-776

33. Chadee K, Petri WA, Innes DJ, Ravdin JI: Rat and human colonic mucins bind to and inhibit adherence lectin of Entamoeba histolytica. J Clin Invest 1987, 80:1245-1254

34. Moncada D, Keller K, Ankri S, Mirelman D, Chadee K: Antisense inhibition of Entamoeba histolytica cysteine proteases inhibits colonic mucus degradation. Gastroenterology 2006, 130:721-730 
35. Dharmani $\mathrm{P}$, Leung $\mathrm{P}$, Chadee K: Tumor necrosis factor- $\alpha$ and Muc2 mucin play major roles in disease onset and progression in dextran sodium sulphate-induced colitis. PLoS One 2011, 6:1-13

36. Van der Sluis M, De Koning BA, De Bruijn AC, Velcich A, Meijerink JP, Van Goudoever JB, Büller HA, Dekker J, Van Seuningen I, Renes IB, Einerhand AW: Muc2-deficient mice spontaneously develop colitis, indicating that MUC2 is critical for colonic protection. Gastroenterology 2006, 131:117-129

37. Velcich A, Yang W, Heyer J, Fragale A, Nicholas C, Viani S, Kucherlapati R, Lipkin M, Yang K, Augenlicht L: Colorectal cancer in mice genetically deficient in the mucin Muc2. Science 2002, 295:1726-1729

38. Van Es JH, de Geest N, van de Born M, Clevers H, Hassan BA: Intestinal stem cells lacking the Math1 tumour suppressor are refractory to Notch inhibitors. Nat Commun 2010, 1:1-5

39. Hasnain SZ, Tauro S, Das I, Tong H, Chen AC, Jeffery PL, McDonald V, Florin TH, McGuckin MA: IL-10 promotes production of intestinal mucus by suppressing protein misfolding and endoplasmic reticulum stress in goblet cells. Gastroenterology 2013, 144:357-368

40. Das I, Png CW, Oancea I, Hasnain SZ, Lourie R, Proctor M, Eri RD, Sheng Y, Crane DI, Florin TH, McGuckin MA: Glucocorticoids alleviate intestinal ER stress by enhancing protein folding and degradation of misfolded proteins. J Exp Med 2013, 210:1201-1216

41. Hu S, Ciancio MJ, Lahav M, Fujiya M, Lichtenstein L, Anant S, Musch MW, Chang EB: Translational inhibition of colonic epithelial heat shock proteins by IFN-gamma and TNF-alpha in intestinal inflammation. Gastroenterology 2007, 133:1893-1904

42. Shkoda A, Ruiz PA, Daniel H, Kim SC, Rogler G, Sartor RB, Haller D: Interleukin-10 blocked endoplasmic reticulum stress in intestinal epithelial cells: impact on chronic inflammation. Gastroenterology 2007, 132:190-207

43. Cao SS, Zimmermann EM, Chuang BM, Song B, Nwokoye A, Wilkinson JE, Eaton KA, Kaufman RJ: The unfolded protein response and chemical chaperones reduce protein misfolding and colitis in mice. Gastroenterology 2013, 144:989-1000

44. Ding W, Yang L, Zhang M, Gu Y: Reactive oxygen species-mediated endoplasmic reticulum stress contributes to aldosterone-induced apoptosis in tubular epithelial cells. Biochem Biophys Res Commun 2012, 418:451-456

45. Quan Z, Gu J, Dong P, Lu J, Wu X, Wu W, Fei X, Li S, Wang Y, Wang J, Liu Y: Reactive oxygen species-mediated endoplasmic reticulum stress and mitochondrial dysfunction contribute to cirsimaritin-induced apoptosis in human gallbladder carcinoma GBCSD cells. Cancer Lett 2010, 295:252-259

46. Hamouda HE, Zakaria SS, Ismail SA, Khedr MA, Mayah WW: p53 Antibodies, metallothioneins, and oxidative stress markers in chronic ulcerative colitis with dysplasia. World J Gastroenterol 2011, 17: $2417-2423$

47. Karp SM, Koch TR: Oxidative stress and antioxidants in inflammatory bowel disease. Dis Mon 2006, 52:199-207

48. Malhotra JD, Kaufman RJ: Endoplasmic reticulum stress and oxidative stress: a vicious cycle or a double-edged sword? Antioxid Redox Signal 2007, 9:2277-2293

49. Radermacher KA, Wingler K, Langhauser F, Altenhöfer S, Kleikers P, Hermans JJ, Hrabě de Angelis M, Kleinschnitz C, Schmidt HH: Neuroprotection after stroke by targeting NOX4 as a source of oxidative stress. Antioxid Redox Signal 2013, 18:1418-1427 